\title{
Targeted tumour theranostics in mice via carbon quantum dots structurally mimicking large amino acids
}

\author{
Shuhua Li ${ }^{1,5}$, Wen Su ${ }^{1,5}$, Hao Wu® 1,5, Ting Yuan1, Chang Yuan1, Jun Liu², Gang Deng², Xingchun Gao², \\ Zeming Chen $\mathbb{1}^{2}$, Youmei Bao², Fanglong Yuan', Shixin Zhou ${ }^{3}$, Hongwei Tan', Yunchao Li', Xiaohong Li', \\ Louzhen Fan ${ }^{1 凶}$, Jia Zhu ${ }^{1 凶}$, Ann T. Chen ${ }^{4}$, Fuyao Liu' ${ }^{2}$, Yu Zhou ${ }^{2}$, Miao Li ${ }^{2}$, Xingchen Zhai ${ }^{2}$ and \\ Jiangbing Zhou $\mathbb{1}^{2,4} \bowtie$
}

\begin{abstract}
Strategies for selectively imaging and delivering drugs to tumours typically leverage differentially upregulated surface molecules on cancer cells. Here, we show that intravenously injected carbon quantum dots, functionalized with multiple paired $\alpha$-carboxyl and amino groups that bind to the large neutral amino acid transporter 1 (which is expressed in most tumours), selectively accumulate in human tumour xenografts in mice and in an orthotopic mouse model of human glioma. The functionalized quantum dots, which structurally mimic large amino acids and can be loaded with aromatic drugs through $\pi$ - $\pi$ stacking interactions, enabled-in the absence of detectable toxicity-near-infrared fluorescence and photoacoustic imaging of the tumours and a reduction in tumour burden after the targeted delivery of chemotherapeutics to the tumours. The versatility of functionalization and high tumour selectivity of the quantum dots make them broadly suitable for tumour-specific imaging and drug delivery.
\end{abstract}

C ancer is one of the most devastating diseases worldwide, with more than 14 million new cases every year. The incidence of cancer is projected to continue to rise, with an estimated 21.7 million new cases by 2030 (ref. ${ }^{1}$ ). In the current clinic, patients with cancer typically receive a combination of treatments, including surgical resection, chemotherapy and radiotherapy, which have substantially increased the overall survival and quality of life of the patients. However, the therapeutic effects of these treatments have been greatly limited by their inability to selectively image and deliver therapeutics to tumours ${ }^{2,3}$. As a result, these treatments are unable to maximize surgical resection or safely deliver chemotherapeutic agents and radiotherapy without inducing significant side effects ${ }^{2,3}$.

To enhance tumour-specific imaging and drug delivery, targeted probes can be engineered through the conjugation of ligands that recognize receptor-like molecules, which are either highly expressed in cancer cells or enriched in the tumour microenvironment $^{2}$. Although promising, this approach is limited ${ }^{4,5}$ by the rarity of molecules that are expressed in cancer cells but not in normal tissues $^{6,7}$ and, therefore, cargo agents can also accumulate in normal organs. Furthermore, cancers are typically heterogeneous across tissues and within tumours and are therefore unlikely to be successfully targeted using a single ligand ${ }^{6,7}$. The ligand-receptor approach is particularly limited for the imaging of brain tumours and the delivery of drugs to them owing to the blood-brain barrier $(\mathrm{BBB})^{8-10}$. A strategy that enables selective imaging of solid tumours and the delivery of drugs to them, regardless of tumour origin and location, is lacking.
One promising approach to tumour-specific imaging and drug delivery leverages specific carrier transporters that are differentially upregulated in cancer cells. Example transporters include large neutral amino acid transporter 1 (LAT1), alanine, serine, cysteinepreferring transporter 2 (ASCT2) and glucose transporters ${ }^{11-14}$. In particular, LAT1 - which mediates the transport of large neutral amino acids-is promising, as it has been shown to be highly expressed in a wide variety of tumours. In normal tissue, LAT1 is expressed in only a few organs, including the placenta, BBB, spleen, testis and colon ${ }^{13}$. LAT1 has previously been targeted for cancer chemotherapy using LAT1 inhibitors, such as 2 -aminobicyclo- $(2,2,1)$ heptane-2-carboxylic acid $(\mathrm{BCH})^{15}$. Unfortunately, $\mathrm{BCH}$-mediated chemotherapy has largely failed owing to a lack of potency and specificity. BCH inhibits all four LATs, including LAT1, LAT2, LAT3 and LAT4, each of which has distinct physiological functions. Effective inhibition of LAT1 requires high doses of $\mathrm{BCH}$, which often induces significant toxicity ${ }^{15}$. LAT1 has also been targeted to enhance the delivery of chemotherapy drugs to tumours. It was shown that conjugation of aspartate enabled LAT1-mediated delivery, improving the accumulation of doxorubicin (DOX) in tumours by threefold to sixfold (ref. ${ }^{16}$ ).

Owing to their excellent biocompatibility, optical properties, drug loading capacity and low toxicity, carbon quantum dots (CQDs) have recently emerged as a promising class of imaging agents and drug carriers for various biomedical applications $s^{17-19}$. However, most of the previously reported CQDs suffer from nonselective interactions with both tumour cells and normal cells ${ }^{17,18}$.

${ }^{1}$ College of Chemistry, Key Laboratories of Theoretical and Computational Photochemistry, and Radiopharmaceuticals, Ministry of Education, Beijing Normal University, Beijing, China. ${ }^{2}$ Department of Neurosurgery, Yale University, New Haven, CT, USA. ${ }^{3}$ Department of Cell Biology and Stem Cell Research Center, School of Basic Medical Sciences, Peking University Health Science Center, Beijing, China. ${ }^{4}$ Department of Biomedical Engineering,

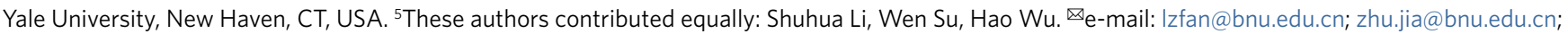
jiangbing.zhou@yale.edu 
<smiles>O=C(O)C[C@H](O)[C@H](O)C(=O)O</smiles>

CA

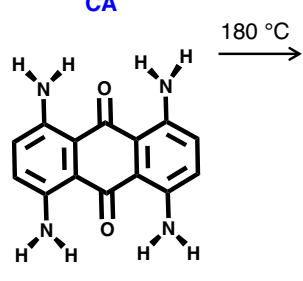

TAAQ

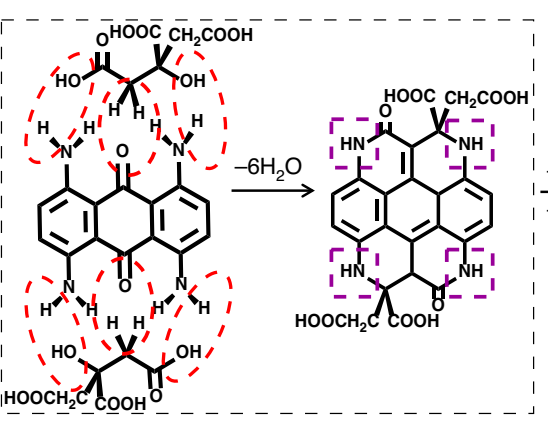

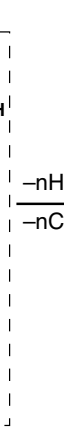

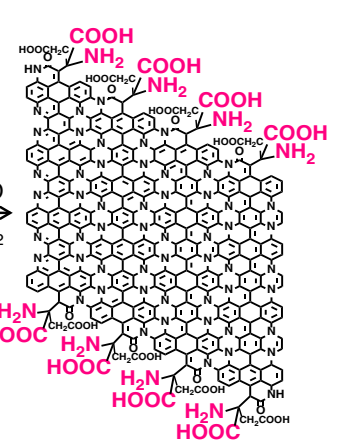

LAAM TC-CQDS

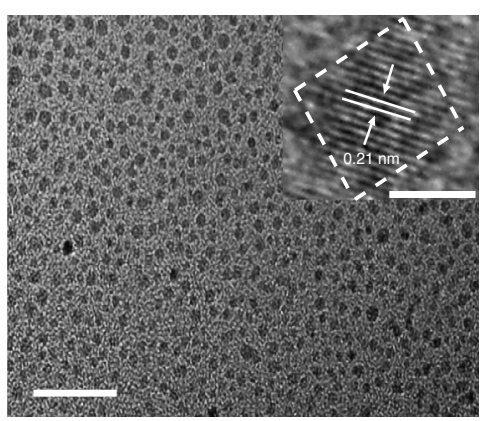

c

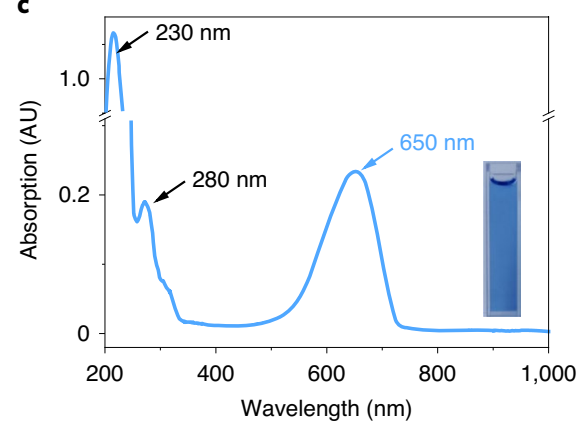

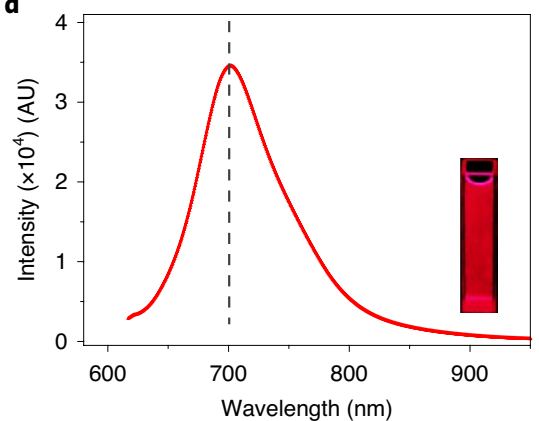

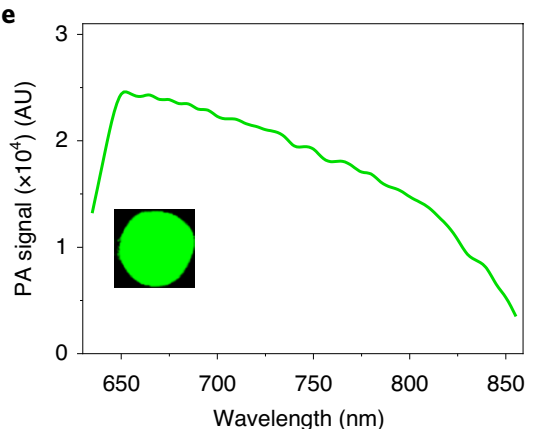

Fig. 1 | Synthesis and characterization of LAAM TC-CQDs. a, Schematic and hypothetical steps (dashed line) of LAAM TC-CQD synthesis. Red dashed circles represent dehydration; purple dashed boxes represent - $\mathrm{NH}$ - groups. b, TEM and HRTEM image (inset) of LAAM TC-CQDs. Scale bars, $10 \mathrm{~nm}$ and $1 \mathrm{~nm}$ (inset). c, Ultraviolet-vis absorption. Inset: appearance of LAAM TC-CQDs under daylight. d, Fluorescence emission spectrum with an excitation wavelength of $600 \mathrm{~nm}$. Inset: appearance of LAAM TC-CQDs under an ultraviolet beam of $365 \mathrm{~nm}$. e, PA signal intensity and imaging (inset) of LAAM TCCQDs. Measurement was performed at a concentration of $2 \mu \mathrm{g} \mathrm{ml}^{-1}$ and at an excitation wavelength of $640-850 \mathrm{~nm}$. AU, arbitrary units.

Here, we report large amino acid mimicking CQDs (LAAM CQDs) for selective imaging and drug delivery to tumours, regardless of the origin and location, but not to normal tissue. LAAM CQDs are a group of CQDs with paired $\alpha$-carboxyl and amino groups on their edge, which trigger multivalent interactions with LAT1. We found that LAAM TC-CQDs-a type of LAAM CQDs that were synthesized using 1,4,5,8-tetraminoanthraquinone (TAAQ) and citric acid (CA) - are capable of near-infrared (NIR) fluorescence and photoacoustic (PA) imaging and can also image and deliver chemotherapy drugs to tumours, including tumours in the brain. In contrast to traditional CQDs, LAAM CQDs have the ability to selectively image and deliver therapeutic agents to tumours. This finding may suggest a promising approach to selectively imaging and delivering therapeutic agents to tumours.

\section{Results}

Synthesis and characterization of LAAM TC-CQDs. LAAM TC-CQDs were synthesized by mixing TAAQ with CA in aqueous solution, followed by hydrothermal treatment at $180^{\circ} \mathrm{C}$ for $2 \mathrm{~h}$ (Fig. 1a). The reactant was purified using silica-gel column chromatography, resulting in a clear blue solution. The procedures for synthesizing LAAM TC-CQD are provided in Supplementary Fig. 1. We demonstrated that this approach ensures that all of the smallmolecular-fragment by-products can be removed (Supplementary Fig. 2). Analysis using transmission electron microscopy (TEM) showed that LAAM TC-CQDs are well dispersed with an average diameter of $2.45 \mathrm{~nm}$ (Fig. 1b, Supplementary Fig. 3). Highresolution TEM (HRTEM) analysis revealed a well-resolved crystal lattice with spacing of $0.21 \mathrm{~nm}$ (Fig. 1b, inset), which is consistent with the (100) lattice fringes of graphene ${ }^{20}$. The high degree of crystallinity was confirmed using Raman spectrum analysis, in which the crystalline $\mathrm{G}$ band at $1,605 \mathrm{~cm}^{-1}$ is stronger than the disordered $\mathrm{D}$ band at $1,365 \mathrm{~cm}^{-1}$, with a G-to-D intensity ratio $\left(I_{\mathrm{G}} / I_{\mathrm{D}}\right)$ of 1.4 (Supplementary Fig. 4). An analysis using atomic force microscopy identified that LAAM TC-CQDs have a typical topographic height of $0.943 \mathrm{~nm}$ (Supplementary Fig. 5), suggesting that most of LAAM TC-CQDs consist of 2-3 graphene layers. Analysis of the X-ray powder diffraction pattern revealed a broad (002) peak centred at $\sim 26^{\circ}$ (Supplementary Fig. 6), confirming the graphene structure of LAAM TC-CQDs.

We determined the molecular structure of LAAM TC-CQDs. First, we determined the amount of amino acid groups on the edge of each LAAM TC-CQD using the classical ninhydrin reaction $^{21}$. After incubation at $60^{\circ} \mathrm{C}$ for $10 \mathrm{~min}$, amino acid groups react with ninhydrin, leading to the formation of the purple-coloured product diketohydrindylidene diketohydrindamine, also known as Ruhemann's purple (RP), which has a maximum absorption at $570 \mathrm{~nm}$. By measuring the absorption of RP, one can therefore calculate the amount of amino acids. The ninhydrin reaction is unique in that the production of RP depends on the amount of primary amino acid groups on the edge but not the structure of backbone, which we confirmed by testing six different amino acids, including aspartic acid, glutamic acid, methionine, phenylalanine, tyrosine and glycine (Supplementary Fig. 7a). We found that the absorption of RP is linearly correlated with the amount of amino acids (Supplementary Fig. 7b). We analysed LAAM TC-CQDs using the ninhydrin reaction and found that treatment with ninhydrin generated a new adsorption peak at $570 \mathrm{~nm}$ (Supplementary Fig. 7c), suggesting the presence of $\alpha$-amino acid groups on the edge. A calculation based on the correlation between amino acids and absorption revealed that there are about eight amino acid groups at the edge of each LAAM TC-CQD. We next determined the chemical 
composition of LAAM TC-CQDs using X-ray photoelectron spectroscopy (XPS), Fourier-transform infrared (FT-IR) spectroscopy and ${ }^{13} \mathrm{C}$ nuclear magnetic resonance (NMR) spectrum. XPS survey spectrum analysis suggested the presence of $\mathrm{C}, \mathrm{N}$ and $\mathrm{O}$ in LAAM TC-CQDs with atomic percentages of $72.43 \%, 12.25 \%$ and $15.32 \%$, respectively (Supplementary Fig. 8). The deconvoluted C1s spectrum revealed that there are three different types of carbon atoms, including graphitic and aliphatic $(\mathrm{C}=\mathrm{C}$ and $\mathrm{C}-\mathrm{C})$, oxygenated $(\mathrm{C}-\mathrm{O}$, $\mathrm{C}=\mathrm{O}$ and $\mathrm{O}-\mathrm{C}=\mathrm{O})$ and $\mathrm{N}$-containing $(\mathrm{C}-\mathrm{N}, \mathrm{C}=\mathrm{N}$ and $\mathrm{N}-\mathrm{C}=\mathrm{O})$ (Supplementary Fig. 9). The deconvoluted N1s spectrum showed three peaks at 399.0, 399.9 and $400.8 \mathrm{eV}$ (Supplementary Fig. 10), which correspond to $\mathrm{C}-\mathrm{N}, \mathrm{N}-\mathrm{H}$ and $\mathrm{C}=\mathrm{N}$, respectively. FT-IR spectrum analysis identified stretching vibrations for $\mathrm{N}-\mathrm{H}\left(3,298 \mathrm{~cm}^{-1}\right.$ and $\left.3,190 \mathrm{~cm}^{-1}\right), C=\mathrm{O}\left(1,720 \mathrm{~cm}^{-1}\right), \mathrm{C}=\mathrm{N} \quad\left(1,624 \mathrm{~cm}^{-1}\right), \mathrm{C}-\mathrm{N}$ $\left(1,387 \mathrm{~cm}^{-1}\right)$ and $\mathrm{C}-\mathrm{O}\left(1,158 \mathrm{~cm}^{-1}\right)$ bonds (Supplementary Fig. 11$)$, suggesting the formation of polyaromatic structures and the presence of free carboxyl and amino groups at the edge of LAAM TC-CQDs. In the ${ }^{13} \mathrm{C}$-NMR spectrum, peaks between $120.0 \mathrm{ppm}$ and $140.0 \mathrm{ppm}$ can be ascribed to aromatic functionalities and conjugated double bonds $(\mathrm{C}=\mathrm{C}$ and $\mathrm{C}=\mathrm{N})$, and peaks at $173.6 \mathrm{ppm}$, $170.2 \mathrm{ppm}$ and $53.3 \mathrm{ppm}$ are associated with $\mathrm{O}-\mathrm{C}=\mathrm{O}, \mathrm{N}-\mathrm{C}=\mathrm{O}$ and $\mathrm{C}-\mathrm{N}$, respectively (Supplementary Fig. 12). The obtained structural information suggests that LAAM TC-CQDs might be formed through two steps. In the first step, under the hydrothermal condition, one molecule of TAAQ reacts with two molecules of $\mathrm{CA}$, resulting a rigid carbon skeleton that consists of three benzene rings fused to four pyridine rings. Subsequently, the skeletons act as building blocks and undergo dehydration and decarboxylation, leading to the formation of LAAM TC-CQDs with high $\mathrm{N}$ doping in the large rigid $\pi$-conjugated structure and free $\alpha$-carboxyl and amino groups at the edge (Fig. 1a). Together with the physical properties of LAAM TC-CQDs, which we determined using TEM and HRTEM, we determined the molecular structure of the majority of LAAM TC-CQDs (Fig. 1a), which is consistent with a theoretical calculation using density functional theory (DFT) simulation (B3LYP/6-31G(d,p)) (Supplementary Fig. 13, Supplementary Table 1). On the basis of the structural information and size distribution, we calculated that the number average molecular mass $(\mathrm{Mn})$ of LAAM TC-CQDs is $6,102.4 \mathrm{~g} \mathrm{~mol}^{-1}$, the weight average molecular mass $(\mathrm{Mw})$ is $62,78.3 \mathrm{~g} \mathrm{~mol}^{-1}$ and the polydispersity index-which is calculated as $\mathrm{Mw} / \mathrm{Mn}-$ is 1.03 . The polydispersity index is close to 1 (monodisperse), suggesting that the LAAM TC-CQDs are highly uniform.

The ultraviolet-visible (ultraviolet-vis) absorption spectrum of LAAM TC-CQDs exhibited a strong characteristic absorption band centred at about $650 \mathrm{~nm}$ (Fig. 1c). Two typical absorption peaks of CQDs at $230 \mathrm{~nm}$ and $280 \mathrm{~nm}$ represent the $\pi-\pi^{*}$ transition of the aromatic $\mathrm{C}=\mathrm{C}$ bond and $\mathrm{n}-\pi^{*}$ transitions of the aromatic $\mathrm{sp}^{2}$ system containing $\mathrm{C}=\mathrm{O}$ and $\mathrm{C}=\mathrm{N}$ bonds, respectively ${ }^{17,22-24}$. Fluorescence characterization was performed using different excitation wavelengths. We found that LAAM TC-CQDs exhibited only one fluorescent emission centre, with a NIR fluorescence emission peak at $700 \mathrm{~nm}$, and the fluorescence emission wavelength was nearly independent of excitation (Fig. 1d, Supplementary Fig. 14). We determined that the absolute fluorescence quantum yield of LAAM TC-CQDs was in the range of $6.8 \%$ to $10.7 \%$ in various solvents using a spectrometer attached to an integrating sphere (Supplementary Table 2).

DFT simulations also confirmed that LAAM TC-CQDs have the local structure for generation of NIR fluorescence. The optimized Cartesian coordinates and main geometric parameters for the ground and excited structures of LAAM TC-CQDs are provided in Supplementary Fig. 13a,b and Supplementary Table 1. The electron delocalization molecular orbital (MO) diagrams of one fluorescence unit obtained from theoretical calculations showed that the energy gap $\left(E_{\mathrm{g}}\right)$ between the highest occupied MO (HOMO) level and the lowest unoccupied MO (LUMO) level is $2.14 \mathrm{eV}$ (Supplementary Fig. $13 \mathrm{c}-\mathrm{e}$ ). The simulation predicted that the excitation wavelength is $580 \mathrm{~nm}$ with an oscillator strength of 0.1092 , and the corresponding fluorescence wavelength is $692 \mathrm{~nm}$ with an oscillator strength of 0.1003 . These values are close to the experimentally measured excitation/emission wavelengths ( $\lambda_{\mathrm{ex}}=600 \mathrm{~nm}, \lambda_{\mathrm{em}}=700 \mathrm{~nm}$; Fig. $\left.1 \mathrm{~d}\right)$.

In addition to their fluorescent properties, we also found that LAAM TC-CQDs have a capacity for PA imaging. The results in Supplementary Fig. 15 and Supplementary Table 3 showed that, when irradiated using a $650-\mathrm{nm}$ laser $\left(0.5 \mathrm{~W} \mathrm{~cm}^{-2}\right)$, LAAM TC-CQDs absorb NIR light, resulting in a thermally induced pressure jump. The resulting ultrasonic waves could be received by an acoustic detector to form PA images ${ }^{25}$. The PA spectra from an agarose gel phantom filled with LAAM TC-CQDs $\left(0-10 \mu \mathrm{g} \mathrm{ml}^{-1}\right)$ were monitored at various excitation wavelengths. The peaks at approximately $650 \mathrm{~nm}$ shown in Fig. 1e and Supplementary Fig. 16 correspond to the observed NIR absorption band of LAAM TC-CQDs shown in Fig. 1c, and the PA signal increased linearly $\left(R^{2}=0.9989\right)$ with a LAAM TC-CQD concentration of $2-10 \mu \mathrm{g} \mathrm{ml}^{-1}$ (Supplementary Fig. 17).

NIR has the advantage of penetrating biological tissues more efficiently than visible light. In contrast to photons, ultrasonic waves are less scattered. PA imaging can therefore overcome the penetration limitations of optical imaging to several centimetres (ref. ${ }^{26}$ ). With this unique NIR fluorescence-PA dual-mode imaging ability, which combines the high contrast of optical imaging with the high spatial resolution of ultrasound, LAAM TC-CQDs represent a promising approach that enable deep-tissue imaging.

LAAM TC-CQDs selectively target cancer cells in vitro and in vivo. We studied the interaction between LAAM TC-CQDs and a large panel of cell cultures, including 27 cancer cell lines of different origin, a side population (SP) of cancer stem-like cells isolated from MDA-MB-231 cells ${ }^{27}$ (Supplementary Fig. 18), 12 patient-derived brain cancer stem cell lines (BCSCs) ${ }^{28,29}$ and 18 non-cancerous cell lines. A detailed list of the cells used in this study is provided in Supplementary Table 4. Using laser confocal scanning microscopy (LCSM), we found that LAAM TC-CQDs penetrated all of the tested cancer cells, including HeLa and A549 cells, regardless of their origin or stemness. By contrast, LAAM TC-CQDs have a limited ability to penetrate non-cancerous cells, such as CCCESF-1 cells. This finding was confirmed using flow-cytometry analysis (Fig. 2a, Supplementary Figs. 19-24). Quantification analysis showed that $\sim 99 \%$ of cancer cells (all except one BCSC line, for which the rate was 95\%) were positive for LAAM TC-CQDs, whereas the rate for non-cancerous cells was limited to $<30 \%$ (average of $13 \%$; Fig. 2b, Supplementary Table 4). LCSM analysis found that a significant fraction of LAAM TC-CQDs entered the nuclei, which was demonstrated by the overlap of LAAM TC-CQD fluorescence and 4,6-diamidino-2-phenylindole (DAPI) fluorescence. We further monitored the intracellular location of LAAM TC-CQDs over time after incubation with HeLa cells. We found that the majority of LAAM TC-CQDs localized on the cell membrane after $1 \mathrm{~h}$ and then entered the cytoplasm. After $6 \mathrm{~h}$, a significant portion of LAAM TC-CQDs had entered the nuclei. By $8 \mathrm{~h}$, the concentration of LAAM TC-CQDs in the nuclei exceeded the concentration of LAAM TC-CQDs in the cytoplasm (Supplementary Fig. 25, Supplementary Video 1). We used the same procedures to monitor the interaction between LAAM TC-CQDs and non-cancerous CCC-ESF-1 cells (Supplementary Fig. 26, Supplementary Video 2). Consistent with our observations using LCSM and flow cytometry, we did not detect interaction between LAAM TC-CQDs and representative non-cancerous CCC-ESF-1 cells throughout the entire time window of $48 \mathrm{~h}$.

We assessed whether LAAM TC-CQDs maintain the observed high degree of tumour specificity in vivo. LAAM TC-CQDs were 
a
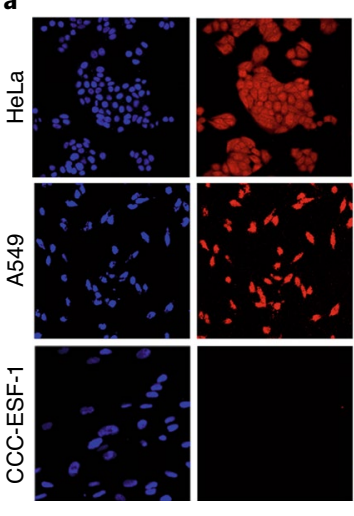

b

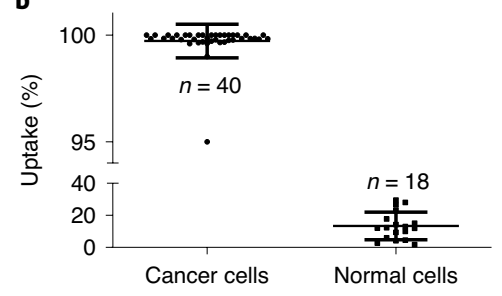

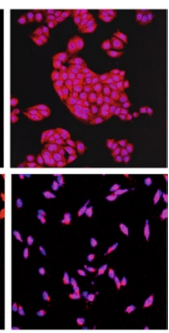

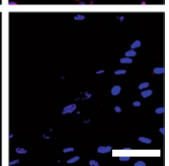

Normal cells

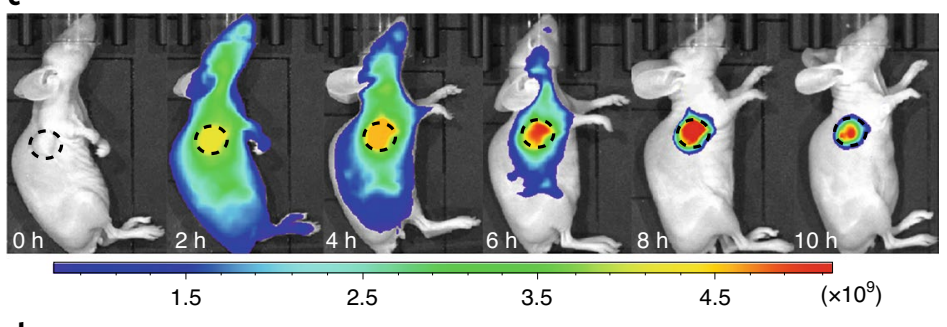

d
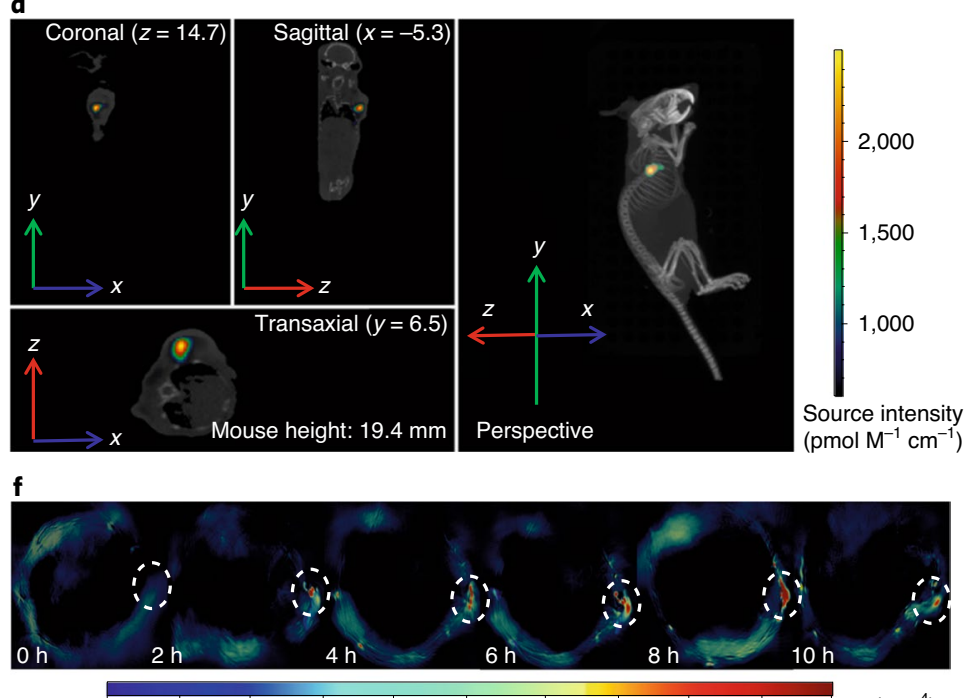
$\left(\mathrm{pmol} \mathrm{M}^{-1} \mathrm{~cm}^{-1}\right.$ ) e

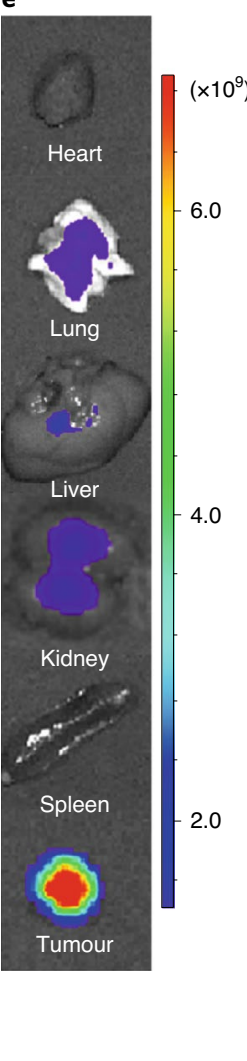

Fig. 2 I LAAM TC-CQDs selectively target cancer cells in vitro and in vivo. a, LCSM images of representative cells, including HeLa cells, A549 cells and non-cancerous CCC-ESF-1 cells, after incubation with LAAM TC-CQDs. Scale bar, $75 \mu \mathrm{m}$. b, Quantification of cellular uptake in cancer and non-cancerous cells using flow cytometry. The rates of cellular uptake for each individual cell type are provided in the Supplementary Information; data are mean \pm s.d. $(P<0.0001)$. c, NIR fluorescence images of a representative mouse bearing a HeLa tumour that received intravenous injection of LAAM TC-CQDs at the

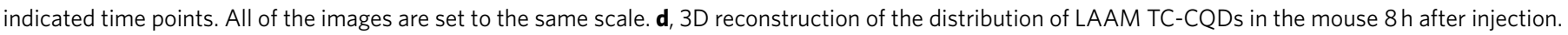
e, Ex vivo NIR fluorescence imaging of the indicated organs and tumour at $8 \mathrm{~h}$ after injection of LAAM TC-CQDs. NIR fluorescence images were captured at excitation/emission wavelengths of $660 \mathrm{~nm} / 740 \mathrm{~nm}$ using an animal optical imaging system. In $\mathbf{c}$ and $\mathbf{e}$, the colour scale indicates intensities in units of radiance (photons $\mathrm{s}^{-1} \mathrm{~cm}^{-2} \mathrm{sr}^{-1}$ ). f, Cross-sectional PA imaging of the indicated tumour (circled) in a representative tumour-bearing mouse that received intravenous injection of LAAM TC-CQDs at the indicated time points. PA imaging was captured at an excitation wavelength of $640-850$ nm at intervals of $5 \mathrm{~nm}$. The colour scale in $\mathbf{f}$ indicates PA signal intensity in arbitrary units. The LAAM TC-CQD concentrations have been standardized for PA signal in vivo (5 $\mathrm{mg} \mathrm{kg}^{-1}$ ).

injected intravenously into mice bearing HeLa tumours at $5 \mathrm{mg} \mathrm{kg}^{-1}$. At $0 \mathrm{~h}, 2 \mathrm{~h}, 4 \mathrm{~h}, 6 \mathrm{~h}, 8 \mathrm{~h}$ and $10 \mathrm{~h}$ after injection, the mice were analysed using fluorescence-PA imaging. Figure $2 \mathrm{c}$ showed that fluorescence in the tumour region gradually increased over time and peaked at $8 \mathrm{~h}$, whereas fluorescence in other regions was not observed. Threedimensional (3D) reconstruction of fluorescence images confirmed that LAAM TC-CQDs selectively imaged tumours but not normal tissues (Fig. 2d, Supplementary Video 3). The high degree of selectivity was validated by ex vivo imaging of isolated organs and tumours, which showed that the fluorescence signal in tumours was substantially greater than that of normal organs (Fig. 2e, Supplementary Fig. 27). Consistent with our finding using fluorescence imaging, the average PA intensity in tumours increased continuously over time until $8 \mathrm{~h}$ after injection, when the PA signal was predominantly concentrated in tumours (Fig. 2f, Supplementary Fig. 28). To exclude the possibility that the observed tumour specificity is unique to HeLa tumours, we evaluated LAAM TC-CQDs in four additional tumour xenografts derived from A549 cells, PANC-1 cells, MCF-7 cells and MD-MBA-231 cells, respectively. We found that LAAM TC-CQDs imaged tumours in all of the tested models, with a kinetics and specificity similar to those observed in the HeLa model (Supplementary Figs. 29 and 30). To further validate the tumour specificity, we evaluated LAAM TC-CQDs in mice bearing a A549 lung carcinoma on the left flank and a HeLa ovarian tumour on the right flank. LAAM TC-CQDs penetrated both tumours with a comparable specificity (Supplementary Figs. 31 and 32).

We studied whether the observed high degree of tumour specificity could be achieved using traditional CQDs. G-CQDs ${ }^{22}$, $\mathrm{Y}-C Q D s^{24}, \mathrm{~B}-\mathrm{CQDs}^{23}$ and $\mathrm{B}, \mathrm{S}-\mathrm{CQDs}{ }^{17}$ were synthesized as examples. We incubated these four previously reported CQDs with a panel of cancer and non-cancerous cells, and we assayed specificity using flow cytometry. As shown in Supplementary Figs. 33 and 34, all of the tested CQDs penetrate both cancer and non-cancerous cells without selectivity. After cell penetration, most of the CQDs were localized in the cytoplasm. G-CQDs were selected and further evaluated in mice bearing HeLa tumours. The experiments were performed according to the same procedures that were used in the LAAM TC-CQDs study. A 3D reconstruction of fluorescence images suggested that, in addition to the tumours, there was fluorescence signal in the liver, lungs and kidneys (Supplementary Fig. 35, Supplementary Video 4). Ex vivo imaging showed that the fluorescence intensities in the lungs and kidneys were substantially 
greater than those in the tumour (Supplementary Fig. 36). We evaluated whether the traditional CQDs could be engineered to reach the degree of tumour specificity observed in LAAM TC-CQDs through conjugation of tumour-targeting ligands. We synthesized G-CQDs with surface conjugation of folic acid (FA) according to our previously published procedures ${ }^{23}$. The resulting CQDs, termed FA-G-CQDs, were evaluated in mice bearing both HeLa and A549 tumours. HeLa tumours, but not A549 tumours, are known to overexpress the FA receptor ${ }^{26,30}$. As expected, we found that FA conjugation enhanced the accumulation of FA-G-CQDs in HeLa tumours, but not in A549 tumours (Supplementary Fig. 37), suggesting that the ligand-conjugation approach primarily enhances the targeting efficiency of CQDs for receptor-expressing tumours. Although the fluorescence signal in HeLa tumours was greater than that of other organs, we observed significant levels of fluorescence signal in the liver, lungs and kidneys, which was confirmed by ex vivo imaging (Supplementary Fig. 38).

We characterized the stability of LAAM TC-CQDs for cancer imaging. The tumour microenvironment is acidic ( $\mathrm{pH} 6.2-6.9$ ), and is further complicated with various molecules, such as proteases and amino acids ${ }^{31,32}$. We therefore characterized the fluorescence stability of LAAM TC-CQDs in $\mathrm{pHs}$ ranging from $\mathrm{pH} 6$ to $\mathrm{pH} 8$ and in the presence of amino acids or matrix metalloproteinase-2 (MMP-2). We found that the fluorescence emission spectrum of LAAM TC-CQDs remained unchanged in all of the tested conditions (Supplementary Fig. 39). We next characterized the photostability of LAAM TC-CQDs after long-term storage or irradiation. None of the tested conditions-including storage at room temperature for 3 months, continuous irradiation with a $500 \mathrm{~W}$ xenon lamp for $12 \mathrm{~h}$ and continuous ultraviolet irradiation at $365 \mathrm{~nm}$ for $5 \mathrm{~h}-$ notably altered the fluorescence emission spectrum (Supplementary Figs. 40 and 41a). Finally, we assessed the impact of long-term incubation in blood serum on the size and fluorescence emission of LAAM TC-CQDs. We found that both the diameter and the fluorescence emission intensity of LAAM TC-CQDs did not change over $30 \mathrm{~d}$ after incubation in blood serum (Supplementary Fig. 42).

Taken together, these data demonstrate that LAAM TC-CQDs have a unique ability to selectively interact with cancer cells, regardless of the origin of the cancer cells, but have a limited ability to interact with non-cancerous cells. As a result of their unique NIR fluorescence-PA dual-mode imaging ability, LAAM TC-CQDs enable in vivo imaging of tumours with minimal background. We found that LAAM TC-CQDs have a level of stability that is suitable for cancer imaging.

LAT1 mediates the internalization of LAAM TC-CQDs into cancer cells. To investigate the manner in which cells uptake LAAM TC-CQDs, we treated HeLa cells with an excess of Leu, Phe or Gly before adding LAAM TC-CQDs. In contrast to Gly, Leu and Phe are both high-affinity substrates of LAT1 $\left(\right.$ ref. ${ }^{33}$ ). We found that the uptake of LAAM TC-CQDs was substantially inhibited by Leu and Phe but not by Gly (Fig. 3a). On the basis of these observations, we hypothesized that LAAM TC-CQDs penetrate cancer cells through interaction with LAT1. This hypothetical mechanism is supported by six lines of evidence. First, pretreatment with the LAT1 inhibitor BCH considerably reduces the uptake of LAAM TC-CQDs by HeLa cells (Fig. 3a). Quantification using flow cytometry showed that pretreatment with $\mathrm{BCH}$ reduced the cellular uptake rates from $\sim 99 \%$ to $\sim 20 \%$ across all of the tested cell lines, including HeLa, A549, PANC-1, MCF-7, MDA-MB-231 and MDA-MB-231 SP cells (Supplementary Fig. 43). Second, LAT1 knockout, which was generated using clustered regularly interspaced short palindromic repeats (CRISPR)-Cas9 and western blotting, markedly reduced the uptake of LAAM TC-CQDs by HeLa cells by $\sim 80 \%$ (Fig. 3b-d, Supplementary Fig. 44). Third, overexpressing LAT1 in HeLa cells through lentiviral transduction enhanced the cellular uptake of
LAAM TC-CQDs in vitro (Fig. 3e). Fourth, the expression level of LAT1 correlated with the amount of LAAM TC-CQDs that penetrated cells in various cell lines (Fig. 3f, Supplementary Fig. 45, Supplementary Table 5). Consistent with previous reports, the level of LAT1 expression in cancer cells was substantially greater than that in non-cancerous cells $s^{11,13,14}$. Fifth, the overexpression of LAT1 in HeLa tumours enhanced the uptake LAAM TC-CQDs by 4.9-fold in vivo (Fig. $3 \mathrm{~g}$ ). Sixth, in an in vivo competition study, pretreatment with Leu reduced the accumulation of LAAM TC-CQDs in tumours by $42 \%$ (Fig. $3 \mathrm{~h}$ ).

We measured the binding affinity between LAAM TC-CQDs and LAT1 using both purified LAT1 protein and HeLa cells. First, we expressed and purified LAT1 protein and determined the binding affinity using surface plasmon resonance. We found that the dissociation constant $K_{\mathrm{d}}$ for the binding between LAAM TC-CQDs and LAT1 is $2.5 \mu \mathrm{M}$ (Supplementary Fig. 46a). We next determined the binding affinity using live HeLa cells. To measure the total binding, cells were treated with various concentrations of LAAM TC-CQDs. After $60 \mathrm{~min}$, the cells were washed, collected and analysed for uptake on the basis of fluorescence intensities using flow cytometry. To determine the non-specific binding, cells were pretreated with $\mathrm{BCH}$ at $5 \mathrm{mM}$ followed by treatment with LAAM TC-CQD and then analysed using flow cytometry. LAT1-dependent uptake was obtained by subtracting the non-specific binding from the total binding. By fitting the Michaelis-Menten equation to the data using weighted nonlinear least squares (Supplementary Fig. 46b), we determined that the binding affinity $K_{\mathrm{m}}$ was $1.4 \mu \mathrm{M}$, which is comparable to the value determined by surface plasmon resonance.

Next, we investigated whether the internalization of LAAM TC-CQDs is mediated by LAT1-dependent transport or endocytosis. First, we performed docking analysis to identify the part of the LAT1 structure that LAAM CQDs bind to. Using the AutoDock Lamarckian Genetic algorithm, we simulated the interaction between LAAM TC-CQDs and the LAT1-4F2hc (CD98) heteromeric complex ${ }^{34}$. We found that LAAM TC-CQDs bind to LAT1 on the edge through hydrogen bonds formed between the amino acid groups in LAAM TC-CQDs and the Lys 77, Asn 242, Leu 238, Trp 452 and Lys 77 residues in LAT1 (Supplementary Fig. 47a). The rigid $\mathrm{sp}^{2}$-conjugate hydrophobic plane is also involved the binding (Supplementary Fig. 47b). We calculated that the docking energy is $E=-2.07 \mathrm{kcal} \mathrm{mol}^{-1}$. No specific interaction between LAAM TC-CQDs and $4 \mathrm{~F} 2 \mathrm{hc}$ was found, suggesting that $4 \mathrm{~F} 2 \mathrm{hc}$ might not be essential for the LAT1-mediated internalization of CQDs. Furthermore, simulation using AutoDock did not find direct interactions between LAAM TC-CQDs and the internal LAT1 binding pocket. Collectively, these findings suggest that uptake of LAAM TC-CQDs probably does not occur through LAT1-mediated transport. Second, we evaluated the internalization of LAAM TC-CQDs in the presence of endocytic-trafficking inhibitors, including chlorpromazine and genistein, which inhibit clathrin- and caveolin-dependent endocytosis, respectively ${ }^{35}$. $\mathrm{BCH}$ was included as a control. We found that both chlorpromazine and $\mathrm{BCH}$ inhibited LAAM TC-CQD internalization by around $80 \%$. By contrast, genistein did not notably inhibit the uptake of LAAM TC-CQDs (Supplementary Fig. 48). These results suggest that the internalization of LAAM TC-CQDs is mediated by clathrin-mediated endocytosis. Third, we measured the transport of LAAM TC-CQDs in cells by continuous fluorescence monitoring in the presence or absence of $\mathrm{BCH}$. We found that, in the absence of $\mathrm{BCH}$, intracellular fluorescence increased over time for the first $6 \mathrm{~h}$, after which no further significant increase was observed. In the presence of $\mathrm{BCH}$, there was little intracellular fluorescence throughout the entire time course (Supplementary Fig. 49). The kinetics of CQD internalization is consistent with the process of receptor-mediated endocytosis $\mathrm{s}^{36,37}$. Fourth, we measured the change in the intracellular concentration of LAAM TC-CQDs after incubation using LCSM. 


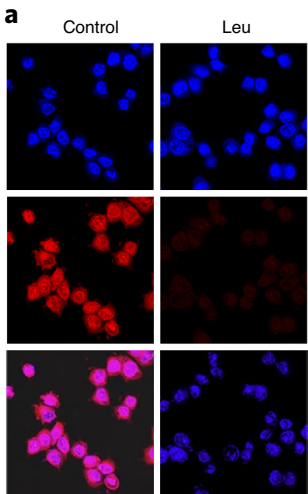

e

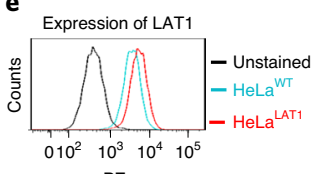

PE

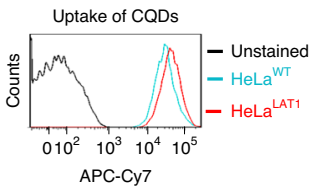

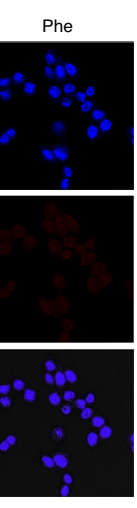

f
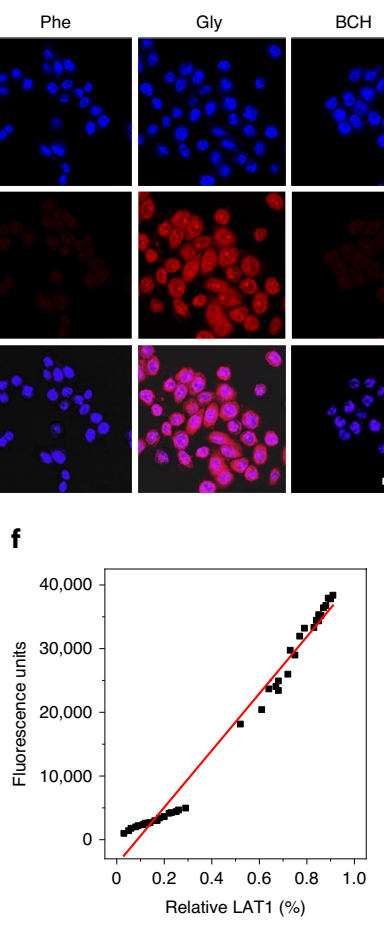

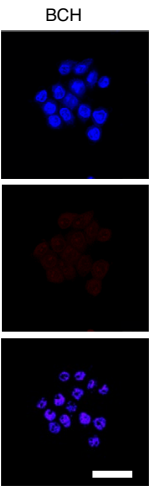

g

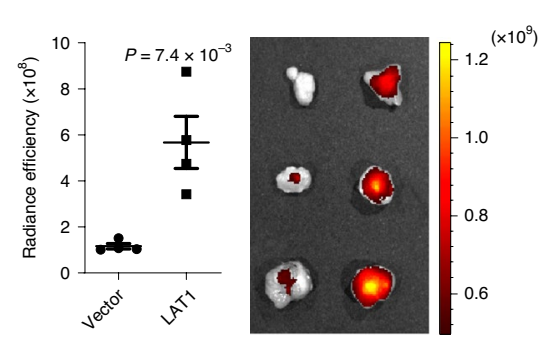

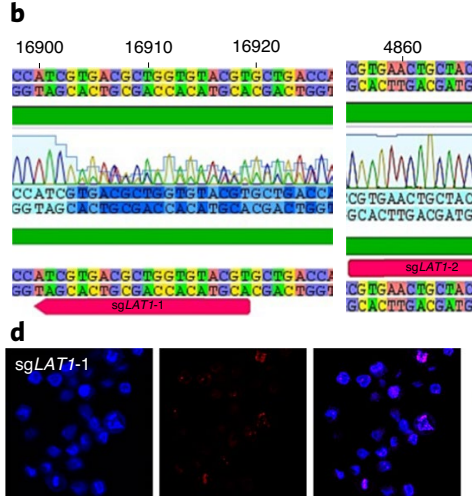

4870

4880
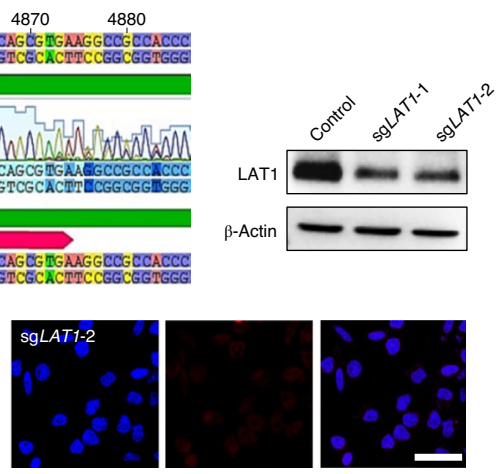

h

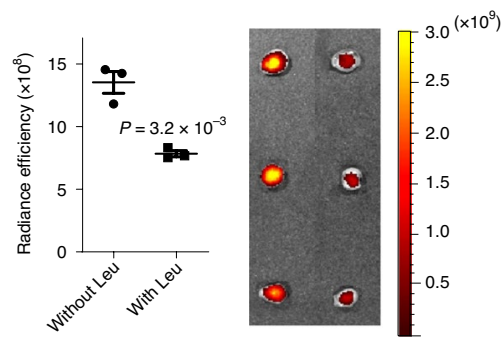

Fig. 3 | The mechanism of the tumour-specific targeting property of LAAM TC-CQDs. a, LCSM images of HeLa cells that were pretreated with Leu, Phe, Gly or BCH at $5 \mathrm{mM}$, followed by incubation with LAAM TC-CQDs. Scale bar, $25 \mu \mathrm{m}$. b-d, Downregulation of LAT1 expression by CRISPR-Cas9 in HeLa cells reduced the cellular uptake of LAAM TC-CQDs (d). The red arrows in $\mathbf{b}$ indicate the sgRNA-targeting sequences. Successful targeting of LAT1 was confirmed using Sanger sequencing (b) and western-blot analysis (c). Full scans of the blots shown in c are provided in Supplementary Fig. 44. LCSM images of cells with downregulation of LAT1 are shown in d (scale bar, $50 \mu \mathrm{m}$ ). e, Overexpression of LAT1 (top) in HeLa cells enhanced the cellular uptake of LAAM TC-CQDs (bottom). PE, phycoerythrin. $\mathbf{f}$, Correlation of LAT1 expression with the cellular uptake of LAAM TC-CQDs. $\mathbf{g}$, Representative images of vector-transfected tumours (left) and LAT1-overexpressing tumours (right), and semi-quantification of LAAM TC-CQDs in mice bearing HeLa tumours $(n=4)$. h, Representative images of tumours isolated from mice without treatment of Leu (left) and tumours isolated from mice treated with Leu at 5 mg

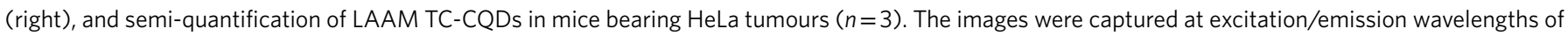
$660 \mathrm{~nm} / 740 \mathrm{~nm}$ using an animal optical imaging system. Intensity was quantified using Living Image v.3.0. In $\mathbf{g}$ and $\mathbf{h}$, the colour scale indicates intensities in units of radiance (photons $\mathrm{s}^{-1} \mathrm{~cm}^{-2} \mathrm{sr}^{-1}$ ). Data are mean \pm s.d. Statistical analysis was performed using two-tailed unpaired Student's $t$-tests.

We found that the concentration of LAAM TC-CQDs within cells was substantially lower than the concentration of LAAM TC-CQDs outside of cells $12 \mathrm{~h}$ after incubation (Supplementary Fig. 50). This observation supports the possibility that LAAM TC-CQDs are probably internalized through LAT1-mediated endocytosis. Finally, we determined whether efflux of LAAM TC-CQDs can be induced by the addition of Leu, a LAT1 substrate. According to the antiport mechanism, the addition of LAT1 substrates should induce efflux of LAAM CQDs if they are internalized through transport. LAAM TC-CQDs were added to HeLa cells at $10 \mu \mathrm{g} \mathrm{ml}^{-1}$. After $12 \mathrm{~h}$, the medium containing LAAM TC-CQDs was removed and replaced with medium containing leucine at $10 \mu \mathrm{g} \mathrm{ml}^{-1}$. After an additional $2 \mathrm{~h}$, the cells were collected and analysed using flow cytometry. We found that external addition of leucine did not induce efflux of LAAM TC-CQDs (Supplementary Fig. 51), suggesting that LAAM TC-CQDs are probably not internalized through transport. Collectively, these data suggest that LAAM TC-CQDs are internalized through LAT1-dependent clathrin-mediated endocytosis.

LAAM TC-CQDs for tumour-specific drug delivery. Major limitations associated with traditional chemotherapy include significant adverse toxicity to normal tissues and intrinsic or acquired drug resistance owing to overexpression of multidrug resistance ATP binding cassette (ABC) transporters. The efficacy of DNAdamaging chemotherapy drugs, which function through interactions with DNA within the nuclei, are often further reduced by their limited ability to penetrate the nucleus ${ }^{38}$. Those limitations can potentially be overcome by using LAAM TC-CQDs as a drug carrier. First, LAAM TC-CQDs have considerable tumour-specific targeting, making it possible to avoid systemic toxicity. Second, owing to their nanoscopic size, LAAM TC-CQDs may not be removed by $\mathrm{ABC}$ transporters ${ }^{39,40}$. Third, LAAM TC-CQDs efficiently penetrate the nucleus, making it possible to maximize the efficacy of DNAdamaging chemotherapy.

We evaluated LAAM TC-CQDs as a drug carrier for the delivery of DNA-damaging chemotherapy drugs. LAAM TC-CQDs have large $\pi$-conjugated structures that enable the loading of aromatic chemotherapy drugs-such as topotecan hydrochloride (TPTC), DOX and hydroxycamptothecin-through a $\pi-\pi$ stacking interaction (Supplementary Fig. 52). We further evaluated TPTC-loaded LAAM TC-CQDs (TPTC-LAAM TC-CQDs), which were synthesized by mixing LAAM TC-CQDs with TPTC overnight followed with extensive dialysis. Successful loading of TPTC was evidenced by the characteristic ultraviolet-vis absorbance peak at $390 \mathrm{~nm}$ that is superimposed on the absorption spectrum of LAAM TC-CQDs (Supplementary Fig. 52a). The slight redshift of the absorption peak of TPTC after loading could be attributed to the $\pi$ - $\pi$-stacking interaction between TPTC and LAAM TC-CQDs. TPTC-LAAM TC-CQDs have dual fluorescence emissions that correspond to TPTC (Supplementary Fig. 53, yellow channel) and LAAM TC-CQDs (Fig. 1d, red channel). We characterized the interaction between TPTC-LAAM TC-CQDs and a panel of cancer cells and 


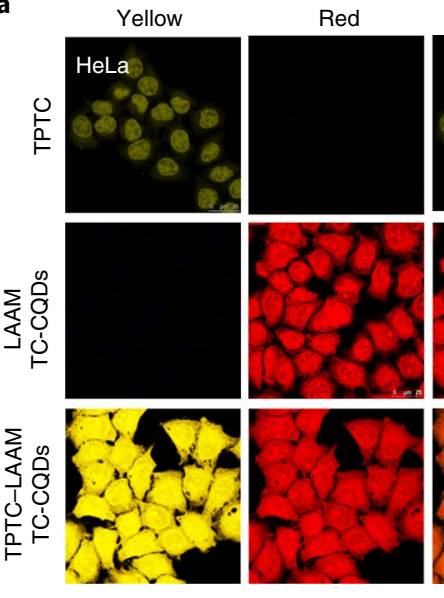

b
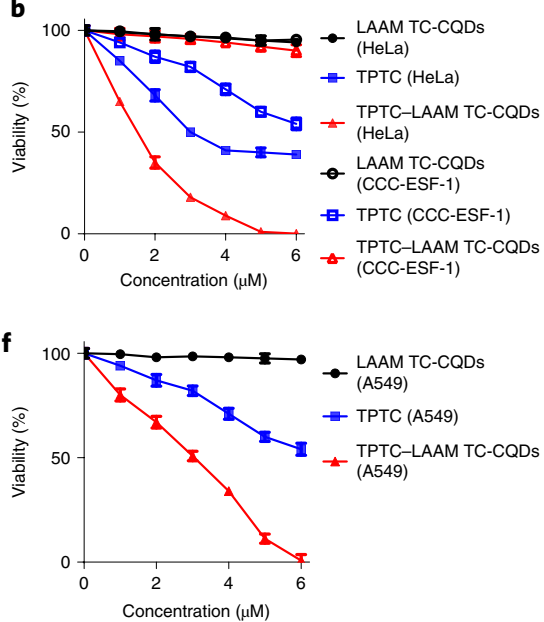

Merge
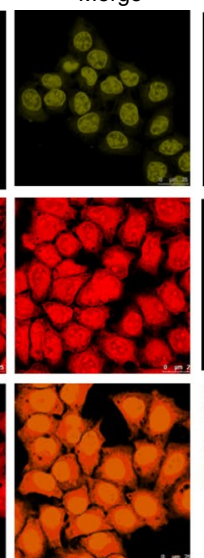

C

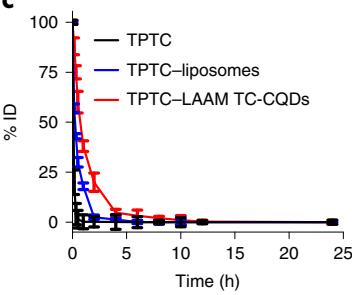

g

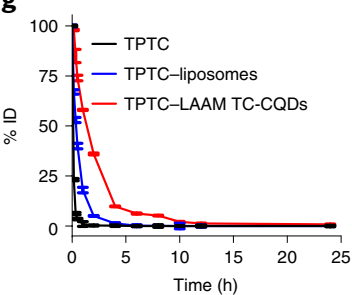

Red
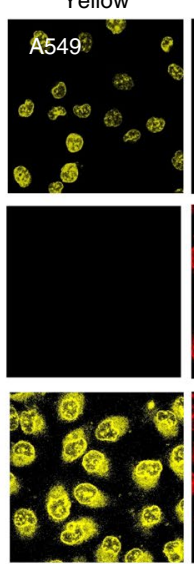
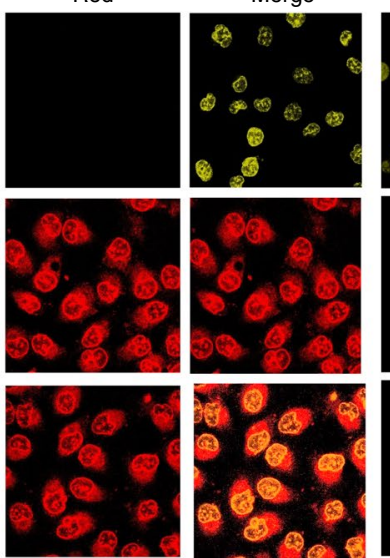

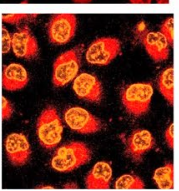

Yellow
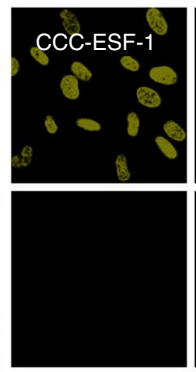

Red
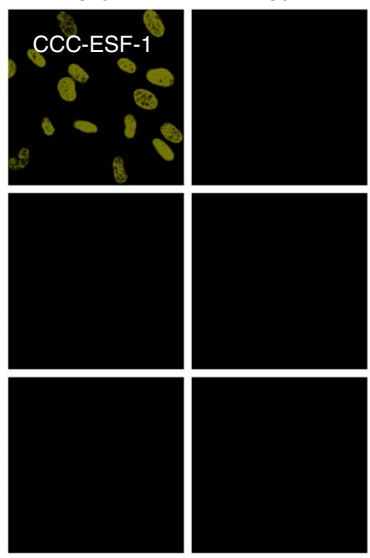

e

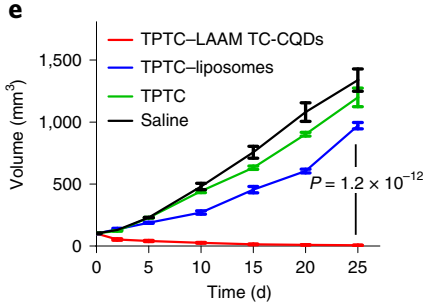

i

h
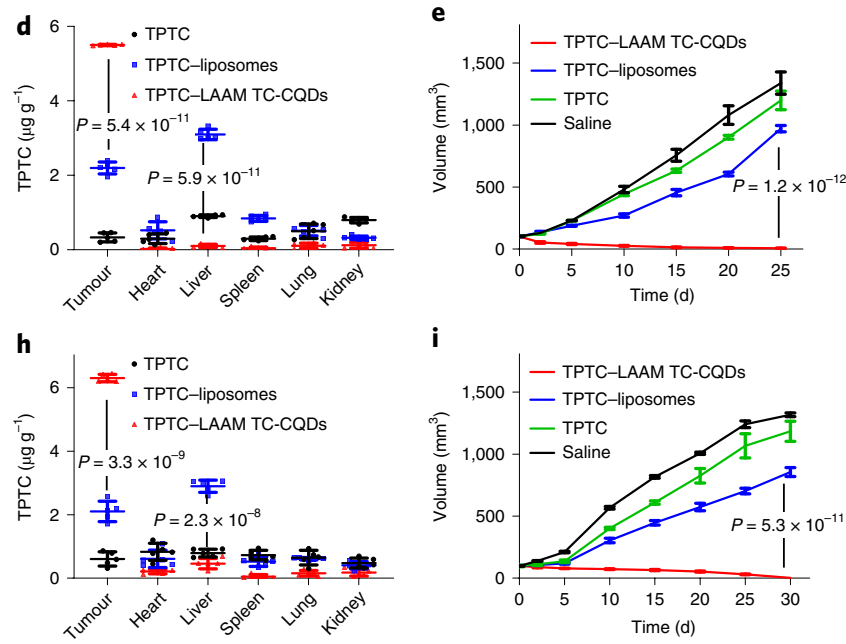

Merge
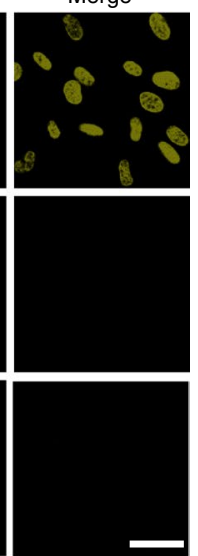

Fig. 4 | LAAM TC-CQDs for tumour-specific drug delivery. a, LCSM images of HeLa, A549 and CCC-ESF-1 cells after incubation with TPTC, LAAM TC-CQDs or TPTC-LAAM TC-CQDs. Scale bar, $25 \mu \mathrm{m}$. b. The viability of HeLa and CCC-ESF-1 cells after treatment with LAAM TC-CQDs, TPTC or TPTC-LAAM TC-CQDs $(n=3)$. c, Changes in the serum concentration of TPTC delivered in the form of free drug or with TPTC-LAAM TC-CQDs over time in mice bearing HeLa tumours $(n=5)$. Data are percentage of total injected dose (\% ID). d, The biodistribution of TPTC and TPTC-LAAM TC-CQDs in the indicated organs isolated from mice bearing HeLa tumours $(n=5)$. e, Changes in tumour volume over time in HeLa-tumour-bearing mice that received the indicated treatments $(n=5)$. f, The viability of A549 cells after treatment with LAAM TC-CQDs, TPTC or TPTC-LAAM TC-CQDs ( $n=3$ ). $\mathbf{g}$, Changes in the serum concentration of TPTC delivered in form of free drug or with TPTC-LAAM TC-CQDs over time in mice bearing A549 tumours ( $n=5$ ). $\mathbf{h}$, The biodistribution of TPTC and TPTC-LAAM TC-CQDs in the indicated organs isolated from mice bearing A549 tumours ( $n=5$ ). $\mathbf{i}$, Changes in tumour volume over time in A549 tumour-bearing mice that received the indicated treatments $(n=5)$. In $\mathbf{b}$-i, data are mean \pm s.d. Statistical analysis was performed using two-tailed unpaired Student's t-tests.

non-cancerous cells. The TPTC-LAAM TC-CQDs retained the selective penetrability to tested HeLa and A549 cancer cells, and delivery by LAAM TC-CQDs eliminates the penetration of TPTC to non-cancerous CCC-ESF-1cells (Fig. 4a). On the basis of fluorescence intensity, delivery by LAAM TC-CQDs enhanced the accumulation of TPTC in the nuclei by 4.5-fold and 5.2-fold for HeLa and A549 cells, respectively (Supplementary Fig. 54).

Next, we characterized TPTC-LAAM TC-CQDs for the treatment of HeLa tumours. Delivery by LAAM TC-CQDs markedly enhanced the toxicity of TPTC to HeLa cells but reduced its toxicity to non-cancerous cells (Fig. 4b). At a concentration equivalent to $5 \mu \mathrm{g} \mathrm{ml}^{-1}$ TPTC, TPTC-LAAM TC-CQDs killed all HeLa cells but did not exhibit toxicity to non-cancerous CCC-ESF-1 cells. By contrast, free TPTC at the same concentration inhibited both cells at a comparable efficiency. The reduced toxicity to normal cells is probably because LAAM TC-CQDs, along with the cargo TPTC, cannot penetrate normal cells (Fig. 4a). Without the ability to penetrate cells, TPTC cannot exert its toxicity, as it functions in the nuclei. We determined the pharmacokinetics of TPTCLAAM TC-CQDs in mice bearing HeLa tumours. For comparison, TPTC-loaded liposomes-designated as TPTC-liposomes-were synthesized using a molar ratio of 56:39:5 HSPC:cholesterol:PEG 2000-DSPE (Supplementary Fig. 55). The same lipid composition was used in several FDA-approved liposomal formulations, including Doxil ${ }^{41}$. TPTC-LAAM TC-CQDs and control free TPTC or TPTC-liposomes were administered intravenously at a dose equivalent to $2 \mathrm{mg} \mathrm{kg}^{-1}$ TPTC. The blood was collected at various time points. TPTC in the plasma was quantified using high-performance liquid chromatography. As shown in Fig. 4c, delivery by LAAM TC-CQDs substantially enhanced the blood circulation and bioavailability of TPTC; the half-life of TPTC increased from $5.4 \mathrm{~min}$ to $40.1 \mathrm{~min}$ and the area under the curve (AUC) increased from 13.4 to 138.1. By contrast, the half-life and AUC of TPTCliposomes were $11.8 \mathrm{~min}$ and 55.1, respectively (Fig. 4c). We next 
examined the biodistribution of TPTC in a second cohort of mice that received the same treatment. As expected, free TPTC was widely distributed over the body without specificity. By contrast, TPTC-LAAM TC-CQDs exhibited kinetics similar to the kinetics of LAAM TC-CQDs (Supplementary Fig. 56). By 8h, TPTC could be detected only in tumours. Ex vivo imaging of isolated organs confirmed that LAAM TC-CQDs enabled highly specific delivery of TPTC to tumours (Supplementary Fig. 57). Quantification on the basis of TPTC fluorescence intensity showed that delivery by LAAM TC-CQDs increased the concentration of TPTC in tumours by 16.7 -fold while substantially reducing the accumulation of TPTC in other organs. The amount of LAAM TC-CQDs accumulated in the tumours accounted for $15.3 \%$ of the total injection dose. By contrast, a significant amount of TPTC-liposomes were found in the liver. Delivery through the liposomes increased the concentration of TPTC in tumours by 6.7-fold (Fig. 4d). We assessed TPTC-LAAM TC-CQDs for treatment of HeLa tumours. When tumour volumes reached around $100 \mathrm{~mm}^{3}$, the mice were randomly grouped and received intravenous administration of saline, TPTC $\left(2 \mathrm{mg} \mathrm{kg}^{-1}\right)$, TPTC-liposomes or TPTC-LAAM TC-CQDs (TPTC-liposomes and TPTC-LAAM TC-CQDs at a dose equivalent to $2 \mathrm{mg} \mathrm{kg}^{-1}$ TPTC) at days $1,4,8$ and 12 . At day 27, tumours in the mice receiving TPTC-LAAM TC-CQDs treatment shrank to an undetectable level (Fig. 4e, Supplementary Fig. 58). By contrast, tumours in the mice receiving free TPTC or TPTC-liposomes continuously grew, although at rates that were slower than that of the control mice (Fig. 4e). Tumour growth curves for individual tumour-bearing mice are provided in Supplementary Fig. 59. All of the mice treated with TPTC-LAAM TC-CQDs survived over $50 \mathrm{~d}$. However, the median survival times for mice treated with saline, TPTC and TPTC-liposomes were limited to $25 \mathrm{~d}, 30 \mathrm{~d}$ and $35 \mathrm{~d}$, respectively (Supplementary Fig. 59).

We repeated the characterization studies using A549 tumours. Experiments were performed according to the same procedures as described above. Similar to the findings in HeLa tumours, we found that delivery by LAAM TC-CQDs substantially enhanced the toxicity of TPTC to A549 cells (Fig. 4f), increased the half-life and AUC in blood circulation of TPTC by 5.5 -fold (from $5.9 \mathrm{~min}$ to $32.5 \mathrm{~min}$ ) and 13.9 -fold (from 15.9 to 221.5 ), respectively (Fig. $4 \mathrm{~g}$ ), increased the accumulation of TPTC in tumours by 10.3fold while reducing TPTC in other organs (Fig. 4h), and substantially inhibited tumour progression and prolonged the survival of tumour-bearing mice (Fig. 4i, Supplementary Fig. 59). The overall tumour-delivery efficiency was $15.8 \%$. Consistent with this, we found that treatment with TPTC-liposomes failed to achieve a comparable degree of inhibitory effects or therapeutic benefits (Fig. 4g-i, Supplementary Fig. 59).

TPTC-LAAM TC-CQDs exhibited minimal toxicity to mice. As shown in Supplementary Fig. 60, treatment with TPTC-LAAM TC-CQDs did not induce apparent weight loss throughout the study. By contrast, weight loss was observed in the groups receiving free TPTC or TPTC-liposomes. Consistent with this finding, haematoxylin and eosin analysis of major organs isolated from normal mice receiving TPTC-LAAM TC-CQD treatment did not detect inflammatory infiltration or pathological damage. However, apparent myocardial fibre rupture, diffuse ballooning degeneration of hepatocytes, pulmonary hyperaemia and focal tubular necrosis were found in mice that were treated with free TPTC (Supplementary Fig. 61). We next performed a complete blood count analysis to measure various parameters, including white blood cells, red blood cells, haemoglobin, haematocrit, mean corpuscular volume, mean corpuscular haemoglobin, mean corpuscular haemoglobin concentration, platelets, red cell distribution width, platelet distribution width, platelet volume and thrombocytocrit. We found that, comparable to mice that received saline as a control, mice that received TPTC-LAAM TC-CQD treatment had parameters within the normal ranges (Supplementary Fig. 62), suggesting that TPTCLAAM TC-CQDs are haemocompatible. Serum biochemistry analyses, including alanine aminotransferase, aspartate aminotransferase and alkaline phosphatase tests for hepatic function, and urea nitrogen and creatinine for renal function, did not detect abnormality in TPTC-LAAM TC-CQD-treated mice (Supplementary Fig. 63). We found that-although LAAM TC-CQDs are nonbiodegradable, demonstrated by the unchanged size and fluorescence emission after incubation for $30 \mathrm{~d}$ in serum-containing PBS (Supplementary Fig. 42)-LAAM TC-CQDs can be rapidly metabolized through renal clearance. Seventy-two hours after intravenous administration, $81.6 \%$ of LAAM TC-CQDs were detected in urine and $8.4 \%$ were detected in faeces (Supplementary Fig. 64).

Collectively, these data suggest that LAAM TC-CQDs can be used as a safe carrier for tumour-specific delivery of chemotherapy, and the delivery by LAAM TC-CQDs substantially enhances the therapeutic benefit while reducing the systemic toxicity of cargo therapeutic agents.

LAAM TC-CQDs for brain cancer imaging and treatment. The BBB, which has been a major hurdle for drug delivery to brain tumours ${ }^{8,9}$, is one of few normal tissues in which LAT1 is expressed $^{13,42}$. We propose that LAAM TC-CQDs are capable of penetrating the $\mathrm{BBB}$ and interacting with brain tumours with high specificity. To test this hypothesis, we intravenously injected LAAM TC-CQDs into mice bearing U87 gliomas, which were analysed using NIR fluorescence imaging at various time points. We found that the accumulation of LAAM TC-CQDs in the brain increased over time and peaked at 8-12 h after injection, when the fluorescence signals were predominately identified in the brain (Fig. 5a). Ex vivo imaging of organs and tumours isolated from mice that were euthanized at the $12 \mathrm{~h}$ time point showed that LAAM TC-CQDs efficiently accumulated in brain tumours (Fig. 5b). 3D reconstruction of fluorescence images confirmed that LAAM TC-CQDs preferentially accumulated in tumours, with substantially lower signal in other tissues (Fig. 5c).

We characterized the role of LAT1 by determining whether the inhibition of LAT1 activity reduces the penetration of LAAM TC-CQDs in an in vitro BBB model as well as in vivo in tumour bearing mice. The in vitro BBB model was established by culturing human brain microvascular endothelial hCMEC/D3 cells on the top insert membrane and human astrocyte NHA cells on the bottom membrane of a Transwell culture plate ${ }^{43}$ (Supplementary Fig. 65a). LAAM TC-CQDs were added to the top chamber. Penetrability was determined by quantifying the proportion of LAAM TC-CQDs that penetrated to the bottom chamber. We found that pretreatment with the LAT1 substrate Leu inhibited the penetrability by $36 \%$ (Fig. 5d). Consistent with this, an in vivo competition study showed that pretreatment with Leu reduced the accumulation of LAAM TC-CQDs in brain tumours by $80 \%$ (Fig. 5 e). Taken together, these results suggest that LAT1 plays a critical role in mediating the brain penetration of LAAM TC-CQDs.

We assessed the ability of TPTC-LAAM TC-CQDs to treat U87 gliomas in the brain. Consistent with our findings in HeLa and A549 cells, TPTC-LAAM TC-CQDs demonstrated a substantially greater cytotoxicity to U87 cells compared with the free TPTC (Fig. 5f). Delivery by LAAM TC-CQDs enhanced the delivery of TPTC to tumours by 16.7 -fold (Fig. 5g) and increased the half-life and AUC of TPTC in the blood by 10.1 -fold (from $4.8 \mathrm{~min}$ to $48.6 \mathrm{~min}$ ) and 12.2-fold (from 23.9 to 290.9; Supplementary Fig. 65b), respectively. By contrast, TPTC-liposomes failed to demonstrate comparable enhancement effects (Fig. 5f,g, Supplementary Fig. 65b). We further analysed the therapeutic benefits of TPTC-LAAM TC-CQDs for treating U87 brain tumours. Two weeks after tumour inoculation, mice were randomly grouped and treated with TPTC $\left(2 \mathrm{mg} \mathrm{kg}^{-1}\right)$ or TPTC-LAAM TC-CQDs or TPTC-liposomes (both at a dose 

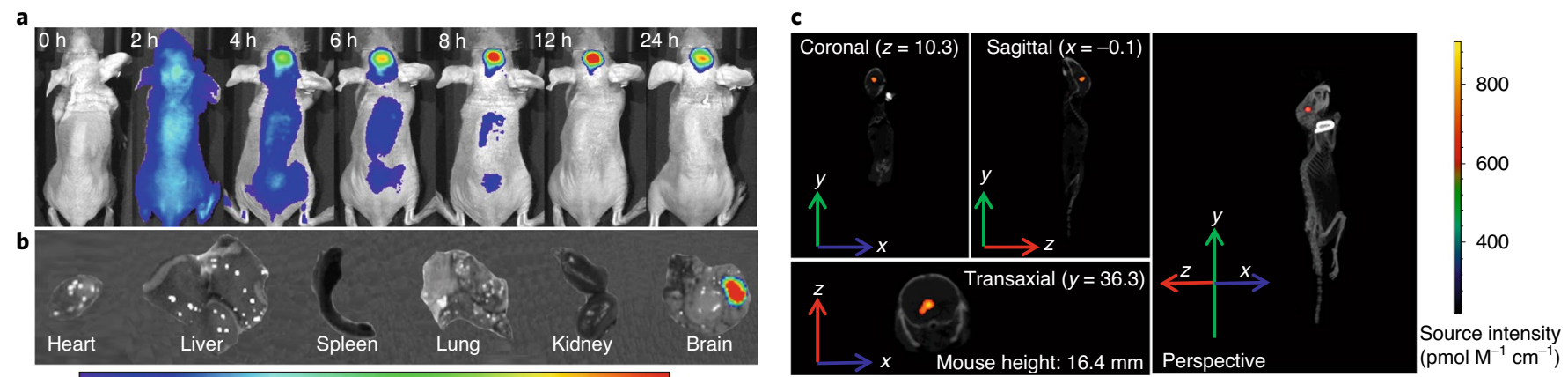

1.5

2.5

3.5

4.5

5.5

$\left(\times 10^{9}\right)$
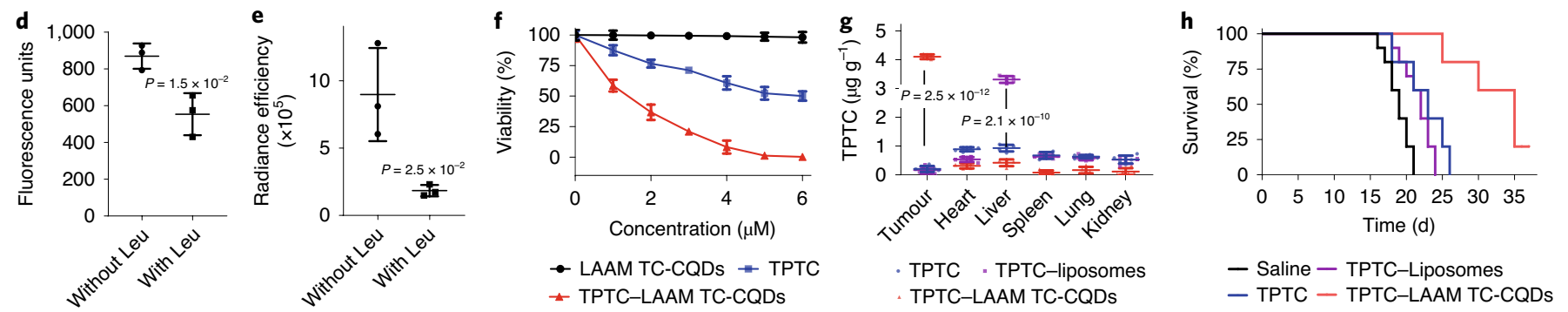

Fig. 5 | The use of LAAM TC-CQDs for brain cancer imaging and treatment. a, NIR fluorescence images of a representative U87-tumour-bearing mouse that received intravenous injection of LAAM TC-CQDs at the indicated time points. All of the images are set to the same scale. $\mathbf{b}$, Ex vivo NIR fluorescence imaging of the indicated organs and tumour $8 \mathrm{~h}$ after injection of LAAM TC-CQDs. c, 3D reconstruction of the distribution of LAAM TC-CQDs in the mouse $8 \mathrm{~h}$ after injection. d, Quantification of LAAM TC-CQDs BBB penetrability without and with treatment of Leu in an in vitro BBB model. e, Semi-quantification of LAAM TC-CQDs in mice bearing intracranial U87 tumours with or without pretreatment of Leu. $\mathbf{f}$, The viability of U87 cells after treatment with LAAM TC-CQDs, TPTC or TPTC-LAAM TC-CQDs. Data are mean \pm s.d. $(n=3)$. $\mathbf{g}$, Changes in serum concentration of TPTC, when delivered in the form of free drug or with TPTC-LAAM TC-CQDs over time in mice bearing intracranial U87 tumours. h, Kaplan-Meier survival analysis revealed that treatment with TPTC-LAAM TC-CQDs significantly enhanced the survival of mice bearing intracranial U87 tumours; $n=10 ; P<0.001$; statistical analysis was performed using the log-rank Mantel-Cox test. In $\mathbf{a}$ and $\mathbf{b}$, the colour scale indicates intensities in units of radiance (photons $\mathrm{s}^{-1} \mathrm{~cm}^{-2} \mathrm{sr}^{-1}$ ). In $\mathbf{d}$, e and $\mathbf{g}$, data are mean \pm s.d. Statistical analysis was performed using two-tailed unpaired Student's $t$-tests.

equivalent to $2 \mathrm{mg} \mathrm{kg}^{-1}$ TPTC), administered three times a week for two weeks. Treatment with TPTC-LAAM TC-CQDs effectively enhanced the survival of tumour-bearing mice to $16 \mathrm{~d}$, which was substantially greater than the survival of the mice that were treated with free TPTC $(3 \mathrm{~d})$ and TPTC-liposomes $(4 \mathrm{~d} ; P<0.001$; Fig. 5h). Furthermore, TPTC-LAAM TC-CQD-treated mice did not decrease in weight over the course of treatment, whereas both free-TPTC-treated and TPTC-liposome-treated mice did (Supplementary Fig. 65c).

LAAM CQDs for tumour-specific imaging and drug delivery. To determine the molecular structure that accounts for the tumour specificity observed in LAAM TC-CQDs, we systematically analysed an array of LAAM TC-CQD analogues. First, we determined whether the carboxyl groups or the amino groups or both are essential. We removed the carboxyl and amino groups on the edge of LAAM TC-CQDs through hydrazine hydrate $\left(\mathrm{NH}_{2}-\mathrm{NH}_{2}\right)$ reduction $^{24}$ and a carbodiimide (EDC)-N-hydroxysuccinimide (NHS) coupling reaction with acetic acid ${ }^{23}$, respectively (Supplementary Figs. 66-68). The resulting $\mathrm{NH}_{2}$ - and $\mathrm{COOH}$-null LAAM TC-CQDs were evaluated in HeLa and CCC-ESF-1 cells. The removal of either carboxyl or amino groups abolished the tumour-specific penetrability of LAAM TC-CQDs, and the resulting CQDs penetrated both cancer and non-cancerous cells without selectivity (Fig. 6a,b). Flowcytometry analysis (Supplementary Fig. 69) revealed that, in contrast to unmodified LAAM TC-CQDs, LAAM TC-CQDs without $\mathrm{NH}_{2}$ groups and $\mathrm{COOH}$ groups had $46 \%$ and $48 \%$ reduced uptake in HeLa cells, respectively, and $414 \%$ and $613 \%$ increased uptake non-cancerous CCC-ESF-1 cells (Fig. 6c). Furthermore, we found that, consistent with previous reports ${ }^{4-46}$, ultraviolet irradiation at $365 \mathrm{~nm}$ for $5 \mathrm{~h}$ deoxidized the surface of CQDs, leading to a significant reduction in the amount of amino acid groups (Supplementary Fig. 41b) and, as a consequence, the remaining LAAM TC-CQDs lost most of their cancer-targeting specificity (Supplementary Fig. 41c). We next tested whether the presence of a carboxyl group and an amino group on the same $\alpha$ position is essential. 2,6-CQDs, which bear the same number of carboxyl and amino groups, were synthesized using 2,6-diaminoanthraquinone (2,6-DAAQ) and $\mathrm{CA}$ and were analysed for their structure and optical properties (Supplementary Figs. 70-75, Supplementary Table 6). All of the carboxyl and amino groups of 2,6-CQDs are located in different positions relative to LAAM TC-CQDs. LCSM and flow-cytometry analysis showed that the altered carboxyl and amino positions results in a complete loss of tumour-specific penetrability; 2,6-CQDs penetrate both HeLa and CCC-ESF-1 cells with comparable efficiency (Fig. 6a-c, Supplementary Fig. 76). We assessed whether the number of paired $\alpha$-carboxyl and amino groups is associated with tumour-specific penetrability. 1,4-CQDs and 1,5-CQDs were synthesized under the same conditions as LAAM TC-CQDs while TAAQ was replaced with 1,4-diaminoanthraquinone (1,4-DAAQ) or 1,5-diaminoanthraquinone (1,5-DAAQ). Both CQDs were analysed for their structure and optical properties (Supplementary Figs. 70-75, Supplementary Table 6). 1,4-CQDs, which are structurally similar to LAAM TC-CQDs but bear half as many paired groups, maintained tumour-specific penetrability (Fig. 6a). However, the penetration efficiency of 1,4 -CQDs was substantially reduced to 
a

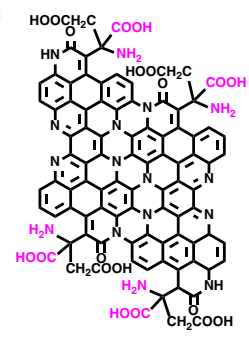

LAAM TC-CQDs
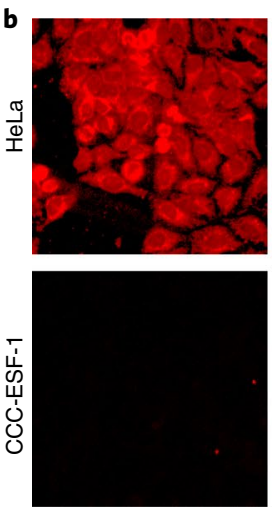

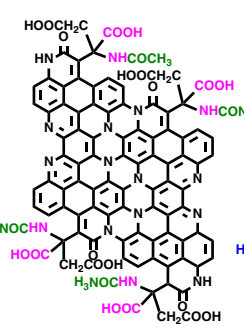

$\mathrm{NH}_{2}$-null LAAM TC-CQDs

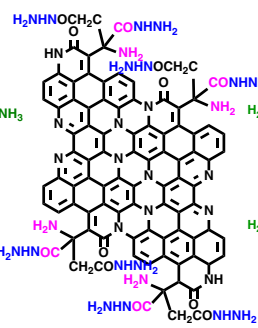

COOH-null LAAM TC-CQDs
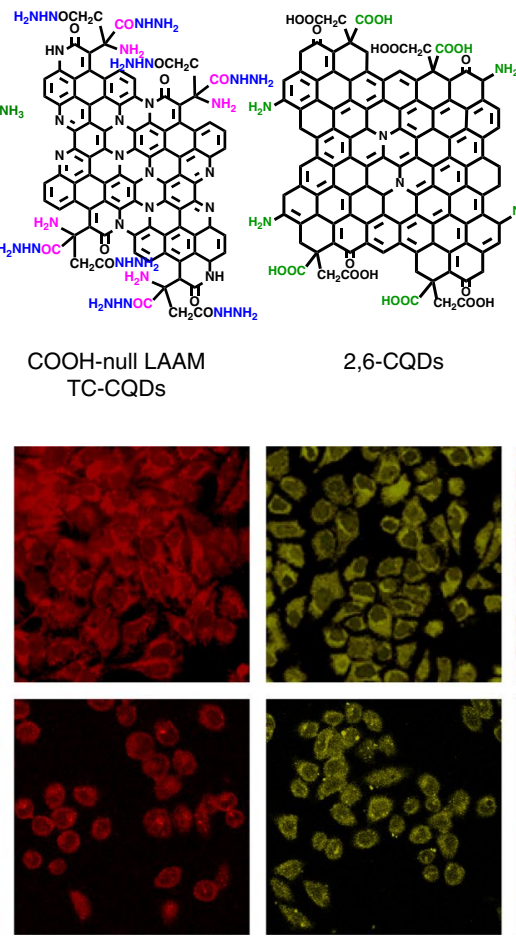

2,6-CQDs

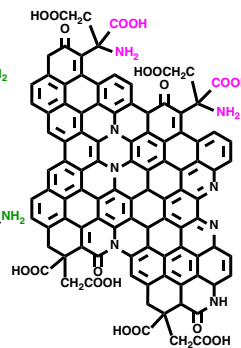

1,4-CQDs
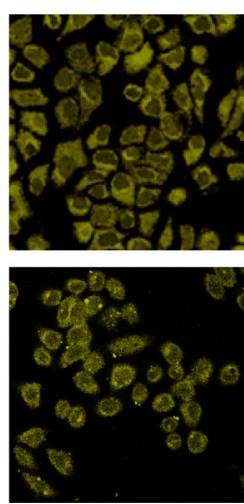
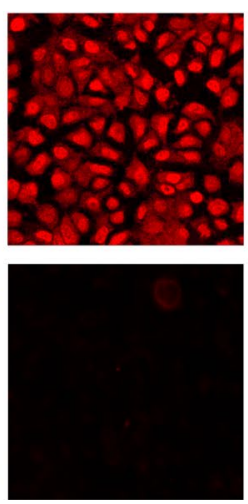

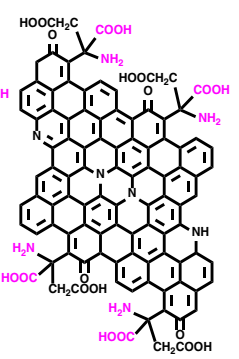

1,5-CQDs
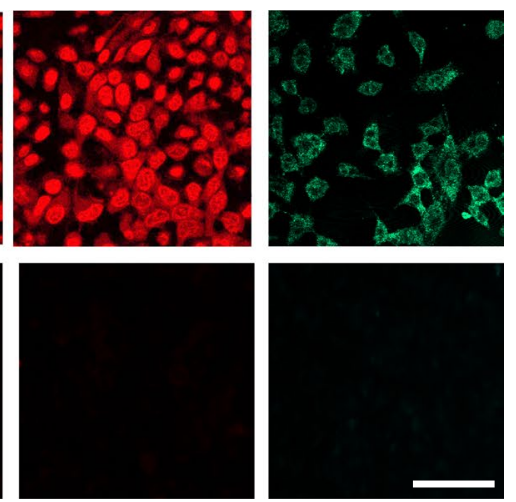

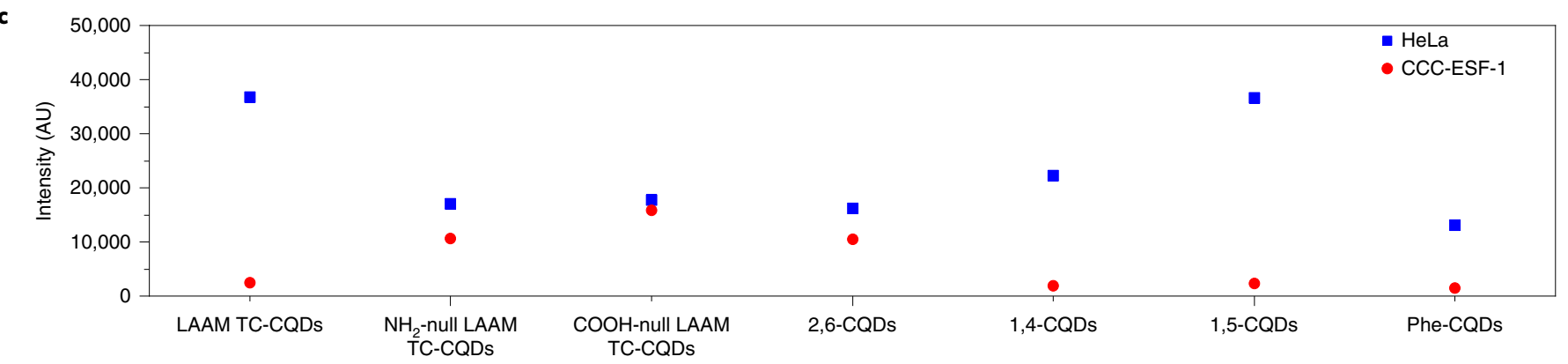

Fig. 6 | Characterization of LAAM CQDs for tumour-specific imaging and drug delivery. a,b, Molecular structures (a) and cell penetrability (b) of LAAM

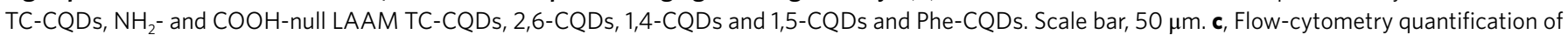
fluorescence intensities in HeLa or CCC-ESF-1 cells after treatment with the indicated CQDs.

$61 \%$ of the penetration efficiency of LAAM TC-CQDs (Fig. 6b,c). 1,5-CQDs, which are also structurally similar to LAAM TC-CQDs and bear the same number of paired groups, penetrated HeLa cells with efficiency that was comparable to LAAM TC-CQDs (Fig. 6a-c). Finally, we determined whether the backbone structure of LAAM TC-CQDs is important for its function. We synthesized Phe-CQDs, which bear paired $\alpha$-carboxyl and amino groups but have a substantially different backbone from LAAM TC-CQDs, using Phe and analysed their structure and optical properties (Supplementary Figs. 77-80). We found that Phe-CQDs maintained the tumour-specific penetrability in HeLa and CCC-ESF-1 cells, although the uptake of Phe-CQDs in HeLa cells was $36 \%$ of that of LAAM TC-CQDs (Fig. 6a-c, Supplementary Fig. 81). The low penetrability of PheCQDs might be attributed to the poor water solubility of PheCQDs, which resulted in low dispersion in solution.

Overall, we found that the high degree of observed tumour specificity is not unique to LAAM TC-CQDs, but to a class of CQDs that bear paired $\alpha$-carboxyl and amino groups. It seems that the anchorage of $\alpha$-carboxyl and amino groups camouflages CQDs as large amino acids, which trigger a LAT1-mediated tumour-specific interaction. We designate CQDs bearing paired $\alpha$-carboxyl and amino groups as LAAM CQDs. Structurally, the location and number of carboxyl and amino groups determine the tumour specificity and efficiency of cell-penetration ability of LAAM TC-CQDs.

\section{Discussion}

Here we developed LAAM CQDs and demonstrated that LAAM CQDs can image and deliver drugs to tumours with different origins and at different locations, including tumours in the brain, with unprecedented specificity and efficiency (Figs. 2, 4 and 5). Through a combination of genetic and pharmacological approaches, we found that the tumour-specific interaction is mediated through LAT1 (Fig. 3). LAAM CQDs have three unique and significant advantages over other imaging and delivery reagents.

First, LAAM CQDs, similar to previously reported CQDs ${ }^{17,18}$, bear intrinsic optical properties for imaging and can therefore be used for tumour imaging without further conjugation of imaging probes $^{47}$. We characterized LAAM TC-CQDs, LAAM CQDs, and found that they enable NIR fluorescence-PA dual-mode imaging, which is optimal for deep-tissue imaging. We demonstrated that LAAM TC-CQDs are capable of imaging tumours with high selectivity (Fig. 2). The high degree of selectivity is probably because LAAM TC-CQDs efficiently penetrate cancer cells but not most normal cells (Figs. 2 and 4). Therefore, after intravenous 
administration, LAAM TC-CQDs are expected to accumulate in tumours and penetrate into tumour cells through interactions with LAT1. By contrast, the CQDs cannot penetrate normal cells and, as a consequence, are eliminated from normal tissues through rapid kidney excretion (Supplementary Fig. 64). Owing to its high specificity to tumours and intrinsic optical properties, LAAM TC-CQDs have great potential to be used for tumour imaging as well as for image-guided surgery.

Second, LAAM TC-CQDs are capable of targeted drug delivery to tumours with a high degree of specificity and efficiency. This cannot be achieved through the conjugation of traditional tumour-targeting ligands, which has also been shown to fail to considerably improve tumour-targeting efficiency ${ }^{4}$. Consistent with these findings, we found that conjugation of ligands enhanced the delivery of CQDs to some tumours, but not others, and did not substantially reduce their non-specific accumulation in normal tissues (Supplementary Fig. 38). Tumour-targeting ligands have previously been tested for LAT1-mediated drug delivery to tumours through conjugation of aspartate ${ }^{16}$. It was found that LAT1 targeting improved the accumulation of DOX in tumours by threefold to sixfold. However, a comparable amount of DOX was also found in the liver. By contrast, we found that delivery by LAAM TC-CQDs enhanced the accumulation of TPTC in tumours by 16.7-fold, while the accumulation of TPTC in the liver was reduced to a barely detectable level $(1.8 \%$ of that in the tumours; Fig. 4d). The observed difference between these two studies may be because LAAM TC-CQDs and aspartate have different affinities with LAT1. We found that the specificity of LAAM CQDs is correlated with the number of paired $\alpha$-carboxyl and amino groups on the edge (Fig. 6). It seems that the local high density of $\alpha$-carboxyl and amino groups triggers a multivalent, strong interaction with LAT1. As a result, delivery by LAAM TC-CQDs, which bear lots of $\alpha$-carboxyl and amino groups, markedly enhances the penetration of TPTC into tumour cells. As most normal tissues do not express LAT1, LAAM TC-CQDs cannot penetrate normal cells and are quickly eliminated from the circulatory system (Fig. 2b). In contrast to LAAM CQDs, aspartate has only one pair of $\alpha$-carboxyl and amino groups and, as a result, the interaction between aspartate-conjugated DOX and LAT 1 could be weak owing to a lack of multivalency. As a consequence, aspartate-conjugated DOX has a low affinity for tumour cells and can non-specifically penetrate normal cells, leading to high accumulation in normal organs ${ }^{16}$.

Third, LAAM CQDs enable the imaging and delivery of drugs specifically to brain tumours. Over the past few decades, we have witnessed significant improvements in the treatments for many types of cancers; however, for solid tumours of the brain, the prognosis remains dismal. The limited progress in the treatment of brain cancer can be largely attributed to the BBB. Although the vasculature of the tumour core tends to be leaky, the BBB is nearly intact in the capillaries that feed the proliferating tumour edge and the surrounding brain tissue and, unfortunately, enhancing drug delivery to brain tumours through traditional ligand-mediated delivery has not been successful ${ }^{8,9}$. The existence of the BBB has also limited the ability to image tumours in the brain, particularly those infiltrating tumour cells located in the region where the BBB is largely intact and where imaging contrast agents cannot be delivered in sufficient quantities ${ }^{48}$. Here we have shown that LAAM CQDsthrough their interaction with LAT1, which is a target expressed in both the BBB and in tumour cells-are capable of imaging and delivering therapeutic agents to tumours in the brain with high specificity and efficiency.

In addition to its application as a probe for tumour imaging and a carrier for drug delivery, LAAM CQD may also be used in the development of LAT1-utilizing prodrugs. LAT1 was successfully targeted for the delivery of several prescription drugs to the brain, such as the antiparkinsonian drug L-dopa and the anticonvulsant drug gabapentin, through a prodrug approach ${ }^{42}$, and has been continuously studied for the delivery of various therapeutic molecules $^{11,33,49}$. More recently, through substantial efforts, several key parameters for the selection of drug candidates and design of LAT1utilizing prodrugs with optimal pharmacokinetics and bioconversion efficiency have been determined ${ }^{50-56}$. This progress lays the foundation for the development of next generation LAT1-utilizing prodrugs and can be applied for synthesizing LAAM CQD-based prodrugs for management of diseases in the central nervous system as well as various cancers.

Overall, LAAM CQDs have a set of properties that are suitable for tumour-specific imaging and drug delivery. Owing to their ability to selectively target tumours regardless of their origin and location, minimal accumulation in most normal tissues, minimal toxicity, intrinsic imaging capacity, ability to load and deliver chemotherapy drugs and ability to penetrate the BBB, LAAM CQDs have potential for translation into clinical applications for imaging and drug delivery to various tumours and diseases of the central nervous system.

\section{Methods}

Cell culture and materials. All cells except for BCSCs were cultured in DMEM or RPMI medium (Invitrogen) supplemented with $10 \%$ fetal bovine serum (FBS; Invitrogen), $100 \mathrm{U} \mathrm{ml}^{-1}$ penicillin and $100 \mu \mathrm{g} \mathrm{ml}^{-1}$ streptomycin (Invitrogen) in a $37^{\circ} \mathrm{C}$ incubator containing $5 \% \mathrm{CO}_{2}$. Human BCSCs were enriched and cultured as previously described ${ }^{28,29}$. All of the chemicals were purchased from Sigma-Aldrich unless otherwise noted. The antibodies against LAT1 were purchased from Novus Biologicals.

\section{Materials and reagents. We purchased 1,4-DAAQ (90\%) and 1,5-DAAQ} (97\%) from Alfa. 2,6-DAAQ (97\%, D124645) was supplied by Shanghai Aladdin Biochemical Technology. TAAQ (30\%, 215643), CA (99.8\%, 791725), dichloromethane $(99.5 \%, 02575)$, methanol $(99.9 \%, 34860)$ and the cell counting kit-8 (CCK-8) were purchased from Sigma-Aldrich. The water used throughout all of the experiments was purified using a Millipore system (ULUPURE). All of the $\mathrm{BALB} / \mathrm{c}$ female mice were purchased from the Beijing Laboratory Animal Research Center, and the weight of each mouse was $18-20 \mathrm{~g}$.

Synthesis of LAAM TC-CQDs. First, $0.04 \mathrm{~g}$ CA and $0.03 \mathrm{~g}$ TAAQ were mixed in $10 \mathrm{ml}$ pure water, and then the solution was transferred into poly(tetrafluoroethylene)-lined autoclaves $(25 \mathrm{ml})$. After heating at $180^{\circ} \mathrm{C}$ for $2 \mathrm{~h}$ and cooling down to room temperature, a blue suspension was obtained. The crude product was then purified with a silica column chromatography (silica gel, 300-400 meshes; chromatographic column, diameter of $5 \mathrm{~cm}$, length of $50 \mathrm{~cm}$ ) using mixtures of dichloromethane and methanol (10:1) as eluents for three rounds in a natural flow rate.

Characterization of CQDs. TEM images were obtained using a JEOL JEM 2100 transmission electron microscope (FEI). Atomic force microscopy images were obtained using a MultiMode V SPM (VEECO) system. X-ray diffraction patterns were performed with an X-ray diffraction system using $\mathrm{Cu}-\mathrm{K} \alpha$ radiation (XRD, PANalytical X'Pert Pro MPD). The Raman spectra were measured using Laser Confocal Micro-Raman Spectroscopy (LabRAM Aramis). XPS was performed using an ESCALab 250Xi electron spectrometer (VG Scientific) using $300 \mathrm{~W}$ Al Koradiation. Ultraviolet-vis absorption and fluorescence spectra were recorded using a UV-2600 spectrophotometer and a PerkinElmer-LS55 fluorescence spectrometer, respectively. FT-IR spectroscopy was measured using a Nicolet 380 spectrograph. The ${ }^{13} \mathrm{C}$ NMR spectra were recorded at $400 \mathrm{MHz}$ using a Bruker Advance III spectrometer in $\mathrm{CH}_{3} \mathrm{OD}$, with chemical shift values displayed in ppm.

Photothermal effects for LAAM TC-CQDs in aqueous solution. To evaluate the photothermal effects in aqueous solution, aqueous solutions of LAAM TC-CQDs at various concentrations $\left(0-10 \mu \mathrm{g} \mathrm{ml}^{-1}\right)$ were exposed to $650 \mathrm{~nm}$ laser irradiation $\left(0.5 \mathrm{~W} \mathrm{~cm}^{-2}\right)$ with the direction of illumination from the top to the bottom of the cuvette for $5 \mathrm{~min}$. An equivalent amount of pure water with the same laser irradiation was used as a control. Real-time temperature was recorded every $30 \mathrm{~s}$ using an infrared thermal camera.

Computational methods. The ground state and the first excited state of one fluorescence unit of CQDs were obtained from theoretical calculations using DFT $(\mathrm{B} 3 \mathrm{LYP} / 6-31 \mathrm{G}(\mathrm{d}))$. The geometric parameters of the ground state were optimized and verified at the B3LYP/6-31G(d) level and the geometric parameters of the first excited state were optimized with TD-B3LYP/6-31G(d). 
Flow cytometry. MDA-MB-231 SP cells were sorted according to our previously reported methods ${ }^{27}$. In brief, cells that were collected at about $85 \%$ confluence were resuspended in RPMI-1640 medium supplemented with $2 \%$ FBS at a density of $1 \times 10^{6}$ cells per $\mathrm{ml}$ and incubated with Hoechst 33342 at a concentration of $5 \mu \mathrm{g} \mathrm{ml} \mathrm{l}^{-1}$ at $37^{\circ} \mathrm{C}$. After $90 \mathrm{~min}$, the cells were suspended in cold PBS at a concentration of $1 \times 10^{6}$ cells per ml, filtered through a cell strainer $(40 \mu \mathrm{m})$ to remove cell aggregates, stained with $1 \mu \mathrm{g} \mathrm{ml}^{-1}$ propidium iodide (PI), and analysed and sorted using a FACSDiva system (BD Biosciences). To characterize SP cells, freshly sorted SP cells were suspended in cold PBS and stained with anti-human CD44-FITC and CD24-PE or their appropriate isotype controls on ice for $30 \mathrm{~min}$. The cells were washed three times with cold PBS, resuspended in $400 \mathrm{ml}$ of cold PBS and analysed using a FACScan flow cytometer (BD Biosciences). To characterize the interaction between CQDs and cells, selected cells were placed on a 6-well plate and treated with LAAM TC-CQDs at $10 \mu \mathrm{g} \mathrm{ml}^{-1}$. Cells that were not treated with CQD were used as controls. After $12 \mathrm{~h}$, the cells were washed with fresh medium, trypsinized, resuspended in PBS with $0.5 \%$ FBS and analysed using a BD FACSCalibur (BD Biosciences). Data were analysed using FlowJo v.7.6.

Confocal laser scanning microscopy imaging. To determine the uptake of CQDs, cells were placed on glass chamber slides and treated with LAAM TCCQDs at $10 \mu \mathrm{g} \mathrm{ml}^{-1}$. After $8 \mathrm{~h}$, cells were washed with PBS twice and fixed with $4 \%$ polyformaldehyde for $30 \mathrm{~min}$ at room temperature, followed by addition of DAPI for cell nuclei staining. Finally, the slides were washed three times, fixed, sealed with cover glasses and imaged with excitation/emission of $561 \mathrm{~nm} / 700 \mathrm{~nm}$ using a confocal laser scanning microscope (Leica TCS-SP8). To determine the effects of Leu, Phe, Gly or BCH on the uptake of CQDs, HeLa cells were placed and treated with Leu, Phe, Gly or BCH. Then, $4 \mathrm{~h}$ later, LAAM TC-CQDs were added to the cells. After an additional $8 \mathrm{~h}$, the cells were washed, fixed and imaged.

PA imaging of LAAM TC-CQDs. LAAM TC-CQDs with different concentrations $\left(0,2,4,6,8\right.$ and $\left.10 \mu \mathrm{g} \mathrm{ml}^{-1}\right)$ were added to agarose tubes $\left(37^{\circ} \mathrm{C}\right)$ and scanned using a PA imaging instrument (iTheraMedical; mode, MOST inVision 128; excitation wavelength ranged from $640-850 \mathrm{~nm}$ with $5 \mathrm{~nm}$ intervals). The PA signal was recorded.

Cellular toxicity tests. Cells were plated into a 96-well plate at a density of $1 \times 10^{4}$ cells per well and treated with LAAM TC-CQDs, TPTC or TPTC-LAAM TCCQDs at various concentrations. After $12 \mathrm{~h}$, the cultured medium was removed and washed with PBS. Then, $100 \mu \mathrm{l}$ of fresh medium containing $10 \mu \mathrm{l} \mathrm{CCK-8} \mathrm{(Sigma-}$ Aldrich) was added to each well. After $2 \mathrm{~h}$ incubation at $37^{\circ} \mathrm{C}$, the absorbance at $450 \mathrm{~nm}$ was measured using a microplate reader.

Animal studies. The research described in this manuscript is compliant with all of the relevant ethical regulations regarding animal research. The animal studies were performed according to protocols that were approved by the Animal Use Committees at Beijing Normal University and Yale University. For in vivo imaging, mice were anaesthetized with isoflurane using an XGI-8 Gas Anaesthesia System (PerkinElmer). Specifically, the mice were placed individually into an induction chamber. Anaesthesia was induced with 5\% isoflurane (Henry Schein) in 100\% oxygen with a delivery rate of $51 \mathrm{~min}^{-1}$ until loss of righting reflex, and then maintained with isoflurane in $100 \%$ oxygen with a flow of $1.5-21 \mathrm{~min}^{-1}$

NIR fluorescence imaging of LAAM TC-CQDs in vivo. Female BALB/c nude mice were maintained in a sterile environment and used for in vivo imaging of LAAM TC-CQDs. To establish mice bearing HeLa tumours, $2 \times 10^{6} \mathrm{HeLa}$ cells were prepared and then subcutaneously inoculated into female BALB/c mice. When the tumour volumes reached about $100 \mathrm{~mm}^{3}$, LAAM TC-CQDs $\left(5 \mathrm{mg} \mathrm{kg}^{-1}\right)$ were intravenously administered into mice. NIR fluorescence and CT images were captured at $1 \mathrm{~h}, 2 \mathrm{~h}, 4 \mathrm{~h}, 6 \mathrm{~h}, 8 \mathrm{~h}$ and $10 \mathrm{~h}$ at excitation/emission wavelengths of $660 \mathrm{~nm} / 740 \mathrm{~nm}$ using an animal optical imaging system (IVIS SpectrumCT, PerkinElmer). For 3D reconstruction, the NIR fluorescence and CT images were co-registered using the fiducial tool in the SpectrumCT Living Image software. Using the FLIT program included in the same software, 3D images were automatically generated. The parameters for NIR fluorescence imaging included: exposure times, $1 \mathrm{~s}$; F/stop, 2; ROI measurements, free drawn; thresholds, $50 \%$; average background ROI, $5.378 \times 10^{7}$. The parameters for $3 \mathrm{D}$ reconstruction imaging included: exposure times, $1 \mathrm{~s}$; F/stop, 2; excitation/emission wavelengths, $660 \mathrm{~nm} / 740 \mathrm{~nm}$; thresholds, $51 \%$. The 3D images were reconstructed using the Living Image software.

PA imaging of LAAM TC-CQDs in vivo. Before intravenous injection of LAAM TC-CQDs, pre-contrast data with excitation wavelengths of $640-850 \mathrm{~nm}$ were obtained. Tumour-bearing mice were treated with LAAM TC-CQDs at $5 \mathrm{mg} \mathrm{kg}^{-1}$. Post-contrast data were acquired at $2 \mathrm{~h}, 4 \mathrm{~h}, 6 \mathrm{~h}, 8 \mathrm{~h}$ and $10 \mathrm{~h}$ after injection. PA images were reconstructed using data acquired from all 128 transducers at each view using a modified back-projection algorithm. Detailed settings for image reconstruction included: method chosen, linear model; FOV, $20 \mathrm{~mm} \times 20 \mathrm{~mm}$; bandpass filters, $50-7.0 \mathrm{MHz}$; optimal speed of sound, $1,541.32 \mathrm{~m} \mathrm{~s}^{-1}$. Detailed settings for fluence correction included: model, light fluence; tissue scattering coefficients, 5. Detailed settings for spectral unmixing included: method, linear regression; wavelengths, $715 \mathrm{~nm}, 730 \mathrm{~nm}, 760 \mathrm{~nm}, 798 \mathrm{~nm}, 800 \mathrm{~nm}$ and $850 \mathrm{~nm}$; chromophores selected, HbO2, Hb, LAAM TC-CQDs. Detailed settings for image filtering and quantitative analysis included: ROI selection, $25 \mathrm{~mm}$; kinetic analysis, PA signal intensities at the tumour area recorded at $0 \mathrm{~h}, 2 \mathrm{~h}, 4 \mathrm{~h}, 6 \mathrm{~h}, 8 \mathrm{~h}$ and $10 \mathrm{~h}$ after CQD administration.

Therapeutic evaluation in tumour-bearing mice. To establish mouse xenografts, tumour cells were subcutaneously inoculated into female $\mathrm{BALB} / \mathrm{c}$ mice. When the tumour volumes reached $\sim 100 \mathrm{~mm}^{3}$, the mice were intravenously administered with saline, TPTC or TPTC-LAAM TC-CQDs $(n=5)$. Changes in tumour volume and body weight were monitored daily. The volume of the tumour was calculated according to the following formula: $V=D \times d^{2} / 2$ where $D$ and $d$ are the longest and shortest diameters of tumour, respectively, measured using a Vernier calliper. Relative tumour volumes were calculated as $V / V_{0}$ where $V_{0}$ is the initial tumour volume when the treatment was started.

Toxicity evaluation in mice. Female BALB/c mice were intravenously administered with TPTC $\left(2 \mathrm{mg} \mathrm{kg}^{-1}, 0.05 \mathrm{ml}\right.$ per mouse $)$ or TPTC-LAAM TCCQDs ( $2 \mathrm{mg} \mathrm{kg}^{-1}$ for TPTC, $0.05 \mathrm{ml}$ per mouse; $\left.n=5\right)$. Mice treated with saline were used as controls. At selected time points, blood samples were collected in heparinized microhematocrit tubes and centrifuged at 3,000 r.p.m. for $10 \mathrm{~min}$. At the end of the study, the mice were euthanized. Major organs, including the heart, liver, spleen, kidney and brain were excised, fixed in formalin and analysed.

Evaluation in brain cancer models. To establish mice bearing brain tumours, nude mice were anaesthetized and positioned on small animal stereotaxic frames. Luciferase-expressing U87 cells $(50,000)$ in $2 \mu \mathrm{l}$ of PBS were injected into the right striatum $2 \mathrm{~mm}$ lateral and $0.5 \mathrm{~mm}$ anterior to the bregma and $3.3 \mathrm{~mm}$ below the dura using a stereotactic apparatus with a UltraMicroPump (UMP3; World Precision Instruments). The weight, grooming and general health of the animals were monitored on a daily basis. Animals were euthanized after either a $15 \%$ loss in body weight or when it was humanely necessary owing to clinical symptoms.

Statistical analysis. For the in vitro experiments, at least three biologically independent experiments were performed unless stated otherwise. Data are presented as the mean \pm s.d. Differences between different groups were compared using unpaired two-tailed Student's $t$-tests. One-way ANOVA analysis was performed to determine the statistical significance of treatment-related changes in survival. Statistical analyses were performed using Prism 8 (GraphPad) and Excel (Microsoft). $P<0.05$ was considered to be statistically significant.

Reporting Summary. Further information on research design is available in the Nature Research Reporting Summary linked to this article.

\section{Data availability}

The main data supporting the results in this study are available within the Article and its Supplementary Information. The raw and analysed datasets that were generated during the study are too large to be publicly shared, but they are available for research purposes from the corresponding authors on reasonable request.

Received: 31 August 2018; Accepted: 20 February 2020; Published online: 30 March 2020

\section{References}

1. Siegel, R. L., Miller, K. D. \& Jemal, A. Cancer statistics, 2016. CA Cancer J. Clin. 66, 7-30 (2016).

2. Kim, S. M., Faix, P. H. \& Schnitzer, J. E. Overcoming key biological barriers to cancer drug delivery and efficacy. J. Control. Release 267, 15-30 (2017).

3. Tringale, K. R., Pang, J. \& Nguyen, Q. T. Image-guided surgery in cancer: a strategy to reduce incidence of positive surgical margins. Wiley Interdiscip. Rev. Syst. Biol. Med. 10, e1412 (2018).

4. Wilhelm, S. et al. Analysis of nanoparticle delivery to tumours. Nat. Rev. Mater. 1, 16014 (2016)

5. Park, K. Facing the truth about nanotechnology in drug delivery. ACS Nano 7, 7442-7447 (2013)

6. Belfiore, L. et al. Towards clinical translation of ligand-functionalized liposomes in targeted cancer therapy: challenges and opportunities. J. Control. Release 277, 1-13 (2018).

7. Bae, Y. H. \& Park, K. Targeted drug delivery to tumors: myths, reality and possibility. J. Control. Release 153, 198-205 (2011).

8. Zhou, J., Atsina, K. B., Himes, B. T., Strohbehn, G. W. \& Saltzman, W. M. Novel delivery strategies for glioblastoma. Cancer J. 18, 89-99 (2012).

9. Deeken, J. F. \& Loscher, W. The blood-brain barrier and cancer: transporters, treatment, and Trojan horses. Clin. Cancer Res. 13, 1663-1674 (2007).

10. Patel, T., Zhou, J., Piepmeier, J. M. \& Saltzman, W. M. Polymeric nanoparticles for drug delivery to the central nervous system. Adv. Drug Deliv. Rev. 64, 701-705 (2012). 
11. Nakanishi, T. \& Tamai, I. Solute carrier transporters as targets for drug delivery and pharmacological intervention for chemotherapy. J. Pharm. Sci. 100, 3731-3750 (2011).

12. Liu, R. et al. GLUT1-mediated selective tumor targeting with fluorine containing platinum(II) glycoconjugates. Oncotarget 8, 39476-39496 (2017)

13. Jin, S. E., Jin, H. E. \& Hong, S. S. Targeting l-type amino acid transporter 1 for anticancer therapy: clinical impact from diagnostics to therapeutics. Expert Opin. Ther. Targets. 19, 1319-1337 (2015).

14. Bodoy, S., Fotiadis, D., Stoeger, C., Kanai, Y. \& Palacin, M. The small SLC43 family: facilitator system 1 amino acid transporters and the orphan EEG1. Mol. Asp. Med. 34, 638-645 (2013).

15. Hayashi, K. \& Anzai, N. Novel therapeutic approaches targeting L-type amino acid transporters for cancer treatment. World J. Gastrointest. Oncol. 9, 21-29 (2017).

16. Wu, W. et al. Aspartate-modified doxorubicin on its N-terminal increases drug accumulation in LAT1-overexpressing tumors. Cancer Sci. 106, 747-756 (2015).

17. Fan, Z., Zhou, S., Garcia, C., Fan, L. \& Zhou, J. pH-responsive fluorescent graphene quantum dots for fluorescence-guided cancer surgery and diagnosis. Nanoscale 9, 4928-4933 (2017).

18. Yuan, F. et al. Shining carbon dots: synthesis and biomedical and optoelectronic applications. Nanotoday 11, 565-586 (2016).

19. Yuan, F. et al. Nitrogen-rich D- $\pi$-A structural carbon quantum dots with a bright two-photon fluorescence for deep-tissue imaging. ACS Appl. Bio. Mater. 1, 853-858 (2018).

20. Peng, J. et al. Graphene quantum dots derived from carbon fibers. Nano Lett. 12, 844-849 (2012).

21. Kendall, P. A. Use of ninhydrin reaction for quantitative estimation of amino groups in insoluble specimens. Nature 197, 1305-1306 (1963).

22. Fan, Z. T. et al. Surrounding media sensitive photoluminescence of boron-doped graphene quantum dots for highly fluorescent dyed crystals, chemical sensing and bioimaging. Carbon 70, 149-156 (2014).

23. Li, S. et al. Exceptionally high payload of the IR780 Iodide on folic acid-functionalized graphene quantum dots for targeted photothermal therapy. ACS Appl. Mater. Inter. 9, 22332-22341 (2017).

24. Zhang, M. et al. Facile synthesis of water-soluble, highly fluorescent graphene quantum dots as a robust biological label for stem cells. J. Mater. Chem. 22, 7461-7467 (2012).

25. Wang, L. V. \& Hu, S. Photoacoustic tomography: in vivo imaging from organelles to organs. Science 335, 1458-1462 (2012).

26. Weber, J., Beard, P. C. \& Bohndiek, S. E. Contrast agents for molecular photoacoustic imaging. Nat. Methods 13, 639-650 (2016).

27. Zhou, J. et al. Activation of the PTEN/mTOR/STAT3 pathway in breast cancer stem-like cells is required for viability and maintenance. Proc. Natl Acad. Sci. USA 104, 16158-16163 (2007).

28. Xiao, Y. et al. Ex vivo dynamics of human glioblastoma cells in a microvasculature-on-a-chip system correlates with tumor heterogeneity and subtypes. Adv. Sci. 6, 1801531 (2019).

29. Zhou, J. et al. Highly penetrative, drug-loaded nanocarriers improve treatment of glioblastoma. Proc. Natl Acad. Sci. USA 110, 11751-11756 (2013).

30. Ren, D., Kratz, F. \& Wang, S. W. Engineered drug-protein nanoparticle complexes for folate receptor targeting. Biochem. Eng. J. 89, 33-41 (2014).

31. Gillies, R. J. The tumour microenvironment: causes and consequences of hypoxia and acidity. Introduction. Novartis Found. Symp. 240, 1-6 (2001).

32. Vaupel, P., Kallinowski, F. \& Okunieff, P. Blood-flow, oxygen and nutrient supply, and metabolic microenvironment of human-tumors-a review. Cancer Res. 49, 6449-6465 (1989).

33. Uchino, H. et al. Transport of amino acid-related compounds mediated by L-type amino acid transporter 1 (LAT1): insights into the mechanisms of substrate recognition. Mol. Pharm. 61, 729-737 (2002).

34. Yan, R., Zhao, X., Lei, J. \& Zhou, Q. Structure of the human LAT1-4F2hc heteromeric amino acid transporter complex. Nature 568, 127-130 (2019)

35. dos Santos, T., Varela, J., Lynch, I., Salvati, A. \& Dawson, K. A. Effects of transport inhibitors on the cellular uptake of carboxylated polystyrene nanoparticles in different cell lines. PLoS ONE 6, e24438 (2011).

36. Ganbold, T., Han, S., Hasi, A. \& Baigude, H. Receptor-mediated delivery of therapeutic RNA by peptide functionalized curdlan nanoparticles. Int. J. Biol. Macromol. 126, 633-640 (2019).

37. Poirier, C., van Effenterre, D., Delord, B., Johannes, L. \& Roux, D. Specific adsorption of functionalized colloids at the surface of living cells: a quantitative kinetic analysis of the receptor-mediated binding. Biochim. Biophys. Acta 1778, 2450-2457 (2008).

38. Cheung-Ong, K., Giaever, G. \& Nislow, C. DNA-damaging agents in cancer chemotherapy: serendipity and chemical biology. Chem. Biol. 20, 648-659 (2013).
39. Yin, J., Deng, X. D., Zhang, J. \& Lin, J. Current understanding of interactions between nanoparticles and ABC transporters in cancer cells. Curr. Med. Chem. 25, 5930-5944 (2018).

40. Yuan, Y. L. et al. Nanoparticle delivery of anticancer drugs overcomes multidrug resistance in breast cancer. Drug Deliv. 23, 3350-3357 (2016)

41. Bulbake, U., Doppalapudi, S., Kommineni, N. \& Khan, W. Liposomal formulations in clinical use: an updated review. Pharmaceutics 9, 12 (2017).

42. Geier, E. G. et al. Structure-based ligand discovery for the large-neutral amino acid transporter 1, LAT-1. Proc. Natl Acad. Sci. USA 110, 5480-5485 (2013)

43. Li, G. et al. Permeability of endothelial and astrocyte cocultures: in vitro blood-brain barrier models for drug delivery studies. Ann. Biomed. Eng. 38, 2499-2511 (2010).

44. Sun, M. et al. Realization of the photostable intrinsic core emission from carbon dots through surface deoxidation by ultraviolet irradiation. J. Phys. Chem. Lett. 10, 3094-3100 (2019).

45. Yuan, F. et al. Engineering triangular carbon quantum dots with unprecedented narrow bandwidth emission for multicolored LEDs. Nat. Commun. 9, 2249 (2018).

46. Yuan, F. et al. Bright multicolor bandgap fluorescent carbon quantum dots for electroluminescent light-emitting diodes. Adv. Mater. 29, 1604436 (2017).

47. Lim, S. Y., Shen, W. \& Gao, Z. Q. Carbon quantum dots and their applications. Chem. Soc. Rev. 44, 362-381 (2015).

48. Mabray, M. C., Barajas, R. F. Jr \& Cha, S. Modern brain tumor imaging. Brain Tumor Res. Treat. 3, 8-23 (2015).

49. Kim, D. K. et al. Characterization of the system $\mathrm{L}$ amino acid transporter in T24 human bladder carcinoma cells. Biochim. Biophys. Acta 1565, 112-121 (2002).

50. Puris, E., Gynther, M., Huttunen, J., Auriola, S. \& Huttunen, K. M. L-type amino acid transporter 1 utilizing prodrugs of ferulic acid revealed structural features supporting the design of prodrugs for brain delivery. Eur. J. Pharm. Sci. 129, 99-109 (2019).

51. Gynther, M. et al. Large neutral amino acid transporter enables brain drug delivery via prodrugs. J. Med. Chem. 51, 932-936 (2008).

52. Gynther, M. et al. Brain uptake of ketoprofen-lysine prodrug in rats. Int. J. Pharm. 399, 121-128 (2010).

53. Huttunen, K. M. et al. L-Type amino acid transporter 1 (LAT1)-mediated targeted delivery of perforin inhibitors. Int. J. Pharm. 498, 205-216 (2016).

54. Peura, L. et al. Large amino acid transporter 1 (LAT1) prodrugs of valproic acid: new prodrug design ideas for central nervous system delivery. Mol. Pharm. 8, 1857-1866 (2011).

55. Puris, E., Gynther, M., Huttunen, J., Petsalo, A. \& Huttunen, K. M. L-type amino acid transporter 1 utilizing prodrugs: how to achieve effective brain delivery and low systemic exposure of drugs. J. Control. Release 261, 93-104 (2017).

56. Gynther, M. et al. Amino acid promoieties alter valproic acid pharmacokinetics and enable extended brain exposure. Neurochem. Res. 41 2797-2809 (2016)

\section{Acknowledgements}

This work was supported by NSFC grants (numbers 21573019 and 21872010 , to L.F.), a NSFC Major Research Plan grant (number 21233003, to L.F.), a NSFC grant (21773016, to J.Zhu) and NIH grants (NS095817 and NS110721, to J.Zhou)

\section{Author contributions}

L.F., J.Zhu, S.L., W.S., H.W. and J.Zhou designed the experiments. S.L., W.S., H.W., T.Y., C.Y., J.L., G.D., F.Y., S.Z., Y.L., X.L., H.T., A.T.C., F.L. and Y.Z. performed the experiments. All of the authors were involved in the analyses and interpretation of data. S.L., L.F. and J.Zhou wrote the paper, with help from the co-authors.

\section{Competing interests}

The authors declare no competing interests.

\section{Additional information}

Supplementary information is available for this paper at https://doi.org/10.1038/ s41551-020-0540-y.

Correspondence and requests for materials should be addressed to L.F., J.Zhu. or J.Zhou. Reprints and permissions information is available at www.nature.com/reprints. Publisher's note Springer Nature remains neutral with regard to jurisdictional claims in published maps and institutional affiliations.

(C) The Author(s), under exclusive licence to Springer Nature Limited 2020 , corrected publication 2022 


\section{natureresearch}

Corresponding author(s): Jiangbing Zhou

Last updated by author(s): Feb 3, 2020

\section{Reporting Summary}

Nature Research wishes to improve the reproducibility of the work that we publish. This form provides structure for consistency and transparency in reporting. For further information on Nature Research policies, see Authors \& Referees and the Editorial Policy Checklist.

\section{Statistics}

For all statistical analyses, confirm that the following items are present in the figure legend, table legend, main text, or Methods section.

n/a Confirmed

$\bigotimes$ The exact sample size $(n)$ for each experimental group/condition, given as a discrete number and unit of measurement

$\bigotimes$ A statement on whether measurements were taken from distinct samples or whether the same sample was measured repeatedly

The statistical test(s) used AND whether they are one- or two-sided

Only common tests should be described solely by name; describe more complex techniques in the Methods section.

Х $\square$ A description of all covariates tested

\ $\square$ A description of any assumptions or corrections, such as tests of normality and adjustment for multiple comparisons

$\triangle \square$

A full description of the statistical parameters including central tendency (e.g. means) or other basic estimates (e.g. regression coefficient)

AND variation (e.g. standard deviation) or associated estimates of uncertainty (e.g. confidence intervals)

$凶 \square$

For null hypothesis testing, the test statistic (e.g. $F, t, r$ ) with confidence intervals, effect sizes, degrees of freedom and $P$ value noted Give $P$ values as exact values whenever suitable.

Х $\square$ For Bayesian analysis, information on the choice of priors and Markov chain Monte Carlo settings

Х $\square$ For hierarchical and complex designs, identification of the appropriate level for tests and full reporting of outcomes

Estimates of effect sizes (e.g. Cohen's $d$, Pearson's $r$ ), indicating how they were calculated

Our web collection on statistics for biologists contains articles on many of the points above.

\section{Software and code}

\section{Policy information about availability of computer code}

Data collection

Data are presented as the means \pm standard deviations. Software used for data collection is listed under 'Data analysis' below. No custom codes were used.

Data analysis

Docking analysis was performed using AutoDock 4.2. Fluorescence image caption, processing and 3D reconstruction were performed using Living Image Version 4.3.1.(64-bit). Photoacoustic image caption and processing were performed using viewMSOT 4.0. Statistical analysis was performed using Prism software version 7.01 (GraphPad Software, Inc., La Jolla, CA). Differences between two groups were compared using the unpaired Student's t-test. Log-rank Mantel-Cox test was performed to determine the statistical significance of treatment related changes in survival. Flow-cytometry analysis was performed using BD FACSDiva software version 6.1.3.

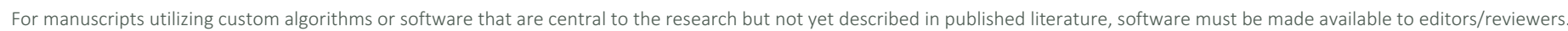
We strongly encourage code deposition in a community repository (e.g. GitHub). See the Nature Research guidelines for submitting code \& software for further information.

\section{Data}

Policy information about availability of data

All manuscripts must include a data availability statement. This statement should provide the following information, where applicable:

- Accession codes, unique identifiers, or web links for publicly available datasets

- A list of figures that have associated raw data

- A description of any restrictions on data availability

The main data supporting the results in this study are available within the paper and its Supplementary Information. The raw and analysed datasets generated during the study are too big to be publicly shared, yet they are available for research purposes from the corresponding authors on reasonable request. 
Please select the one below that is the best fit for your research. If you are not sure, read the appropriate sections before making your selection. $\bigotimes$ Life sciences Behavioural \& social sciences Ecological, evolutionary \& environmental sciences

For a reference copy of the document with all sections, see nature.com/documents/nr-reporting-summary-flat.pdf

\section{Life sciences study design}

All studies must disclose on these points even when the disclosure is negative.

Sample size Sample sizes were determined on the basis of estimates from preliminary experiments and previous similar work, so that reasonable statistical analyses could be conducted.

Data exclusions For the in vitro analyses, we did not exclude any data. For the in vivo experiments, we excluded mice that died due to overexposure to anesthesia.

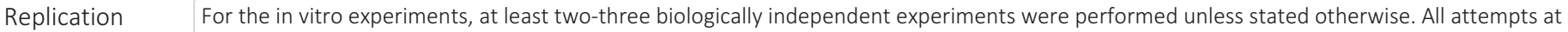
replication were successful. For the in vivo experiments, we excluded mice that died due to overexposure to anesthesia. At least 5 animals were used in the statistical analysis for survival time.

Randomization In the animal studies, mice inoculated with the same batch of tumor cells were randomly grouped into the experimental groups. The experimenter who imaged tumors and recorded survival was blinded to the treatment groups.

Blinding

Key studies, including imaging, flow cytometry and animal tests, were recorded and analysed in a blinded fashion.

\section{Reporting for specific materials, systems and methods}

We require information from authors about some types of materials, experimental systems and methods used in many studies. Here, indicate whether each material, system or method listed is relevant to your study. If you are not sure if a list item applies to your research, read the appropriate section before selecting a response.

\begin{tabular}{l|l} 
Materials \& experimental sys \\
\hline n/a & Involved in the study \\
$\square$ & $\bigotimes$ Antibodies \\
$\square$ & $\square$ Eukaryotic cell lines \\
$\square$ & $\bigotimes$ Palaeontology \\
$\square$ & $\square$ Animals and other organisms \\
$\square$ & $\square$ Clinical data
\end{tabular}

\begin{tabular}{l|l}
\multicolumn{2}{l}{ Methods } \\
\hline n/a & Involved in the study \\
$\square$ & $\square$ ChIP-seq \\
$\square$ & $\square$ Flow cytometry \\
$\square$ & $\square$ MRI-based neuroimaging
\end{tabular}

Antibodies

Antibodies used

LAT1, Cell signaling, Cat.\# 53475, 1:1000, lot: 3.

beta Actin, Biolegend, Cat.\# 643802, lot: B266259

Validation

We confirmed each primary antibody for the species and application specified on the manufacture's website.

\section{Eukaryotic cell lines}

Policy information about cell lines

Cell line source(s)

Cell lines (HeLa, A549, PANC-1, MCF-7, MDA-MB-231, MKN-45, A498, HepG2, HTB-9, EC109, PC-3, SF126, SK-MEL-1, NClH1975, T84, CAL-27, SH-SY5Y, Hep-2, H460, Jurkat, CCC-ESF-1, HUVEC, CCD-1095SK, HL-7702, RWPE-1, HaCaT, Hs578Bst, MCF 10A, BEAS-2B, MRC-5, WISH, HKC, 1301, HLF-a, CCC-HEK-1, CCC-HPE-2, CCC-HIE-2 and HBMSCs) were purchased from ATCC and YMFX Biotech Co., Ltd.

Authentication

The vendors had performed cell authentication.

Mycoplasma contamination

The cell lines were tested for mycoplasma contamination regularly every four weeks via the Mycoplasma Detection Kit (Sigma). All cell lines tested negative for mycoplasma. 


\section{Animals and other organisms}

Policy information about studies involving animals; ARRIVE guidelines recommended for reporting animal research

Laboratory animals

Wild animals

Field-collected samples

Ethics oversight
Female nude mice (BALB/c nu/nu, 5-6 weeks old) were used.

The study did not involve wild animals.

The study did not involve samples collected from the field.

Animal studies were performed according to the protocols approved by the Animal Use Committees at Beijing Normal University and Yale University.

Note that full information on the approval of the study protocol must also be provided in the manuscript.

\section{Flow Cytometry}

\section{Plots}

Confirm that:

\The axis labels state the marker and fluorochrome used (e.g. CD4-FITC).

X The axis scales are clearly visible. Include numbers along axes only for bottom left plot of group (a 'group' is an analysis of identical markers).

$\bigotimes$ All plots are contour plots with outliers or pseudocolor plots.

$\triangle$ A numerical value for number of cells or percentage (with statistics) is provided.

\section{Methodology}

Sample preparation

Instrument

Software

Cell population abundance

Gating strategy
Cells were treated with CQDs. Typically after $4 \mathrm{~h}$ (if otherwise, this is noted in the text), cells were collected, fixed and subjected to flow-cytometry analysis.

BD FACSAria

BD FACSDiva software version 6.1.3

Over 105 cells were counted.

Control cells without treatment were analysed to set up gates.

Tick this box to confirm that a figure exemplifying the gating strategy is provided in the Supplementary Information. 